\title{
Expectation Maximization-Based Passive Localization Relying on Asynchronous Receivers: Centralized versus Distributed Implementations
}

\author{
Weijie Yuan, Nan Wu, Bernhard Etzlinger, Yonghui Li, Senior Member, IEEE, Chaoxing Yan, \\ and Lajos Hanzo, Fellow, IEEE
}

\begin{abstract}
This paper considers a passive localization scenario relying on a single transmitter, several receivers and multiple moving targets to be located. The so-called "passive" targets equipped with RFID reflectors are capable of reflecting the signals from the transmitter to the receivers. Existing approaches assume that the transmitter and receivers are synchronous or quasi-synchronous, which is not always realistic in practical scenarios. Hence, an asynchronous wireless network is considered, where different clock offsets are assumed at different receivers. We propose a centralized expectation maximizationbased passive localization method for asynchronous receivers (EMpLaR) by treating the clock offsets as hidden variables. Thereby, the proposed algorithm makes use of Taylor expansions to arrive at a closed-form maximization. Furthermore, to improve the robustness to link failures and to reduce the energy consumption, we propose a distributed localization approach based on average consensus formulation to locate the target at each receiver. By applying a quadratic polynomial approximation of the function on which consensus has to be reached, both the computational complexity and the communications overhead are significantly reduced. The Cramér-Rao bound of the target location is derived as a benchmark of our proposed algorithms. Our simulation results show that the proposed centralized and distributed EMpLaR algorithms match the Cramér-Rao bound and significantly improve the localization performance compared to the conventional methods.
\end{abstract}

Index Terms-Passive Localization, Expectation Maximization, Asynchronous Receivers, Wireless Sensor Networks, Average Consensus, Quadratic Polynomial Approximation

This work was supported by the "National Science Foundation of China (NSFC)" (Grant No.61571041, 61471037), "A Foundation for the Author of National Excellent Doctoral Dissertation of P. R. China (FANEDD)' (Grant No. 201445) and the Linz Center of Mechatronics (LCM) in the framework of the Austrian COMET-K2 program. L. Hanzo would like to acknowledge the financial support of the European Research Council's Advanced Fellow Award QuantCom and of the EPSRC projects EP/Noo4558/1, EP/PO34284/1(Corresponding author: Nan Wu)

W. Yuan and N. Wu are with the School of Information and Electronics, Beijing Institute of Technology, Beijing, China, (email: \{wjyuan, wunan\}@bit.edu.cn).

B. Etzlinger is with the Institute for Communications Engineering and RF-Systems, Johannes Kepler University, Linz, Austria, (email: bernhard.etzlinger@jku.at).

Y. $\mathrm{Li}$ is with the School of Electrical and Information Engineering, University of Sydney, NSW, Australia (e-mail:yonghui.li@sydney.edu.au).

C. Yan is with the School of Electronics and Computer Science, University of Southampton, SO17 1BJ, UK and is also with the Beijing Research Institute of Telemetry, Beijing, China (email: c.yan@soton.ac.uk).

L. Hanzo is with the School of Electronics and Computer Science, University of Southampton, SO17 1BJ, UK (e-mail:1h@ecs.soton.ac.uk).

Part of this paper has been presented in the Global Communications Conference (GLOBECOM) 2015, San Diego, USA.

Since this paper is based on a complex China, Austria, Australia \& UK collaboration, so researcg data is available.

\section{INTRODUCTION}

Wireless sensor networks (WSNs) have significantly contributed to improving services in contemporary society [1]. Since many applications of WSNs rely on the location information, such as environmental monitoring, emergency rescue and vehicle tracking, the awareness of location has become more and more important and has received much attention [2], [3].

Using radio frequency signals, location related measurements can be obtained by utilizing time of arrival (TOA), angle of arrival (AOA) and received signal strength (RSS) estimates [4]-[6]. AOA-based methods require specific antenna structures, while RSS-based methods suffer from fast fading in wireless propagation scenarios. TOA-based methods can use any antenna setup and the time measurements are robust to channel fading. In what follows we will focus on the family of TOA-based techniques. Depending on the participation of the target (object to be localized), the localization problem can be further classified into "active" and "passive" localization. In active localization, the targets cooperate with other network members and actively emit signals. These signals are used for measuring the inter-node distance, which induces an annulus for possible target location. The intersection of multiple such annuli is then used for estimating the location of the "active" target. By contrast, in passive localization, the signal received from the transmitter is reflected by the passive target and is then received at different receivers. The signal propagation time identifies several ellipses with the foci at the positions of the target and of the different receivers [7]. The intersection of those ellipses can provide location estimate of the "passive" target. The passive targets in WSNs may include animals, unmanned vehicles, people that are not capable of locating themselves. Passive localization has numerous applications such as object monitoring, vehicle tracking relying on opportunistic signals and so forth. ${ }^{1}$

One of the main challenges faced by the family of TOAbased methods is the vital requirement for time synchronization of all nodes in WSNs. Even a low clock offset on the order of tens of nano-seconds would significantly degrade the localization performance. This poses a more stringent timing constraint than that in wireless communications, where

\footnotetext{
${ }^{1}$ A different scenario where the target is non-cooperative but emits arbitrary signals is also termed "passive" [8], [9]. That scenario is not considered in this work.
} 
synchronization accuracy on the order of micro-seconds is often sufficient.

In general, performing synchronization [10]-[13] before localization [14]-[17] can be applied. However, this mechanism increases the communications overhead. Recently, the close relationship between localization and synchronization is unveiled by simultaneously solving the two problems [18][23]. Passive localization in a synchronous network was studied in [24], [25], and in a quasi-synchronous network (all receivers experience the same clock offset) in [26]. In [27], TOA measurements were used for acquiring time difference of arrival (TDOA) estimates, when the receivers are asynchronous. However, due to the noise enhancement imposed, TDOA based methods suffer from inevitable performance degradations. In this contribution, we avoid the generation of TDOA measurements and propose novel algorithms for passive localization relying on asynchronous receivers purely based on TOA measurements.

On the other hand, the aforementioned passive localization methods rely on fusion centers for performing centralized estimation. Therefore, all the measurements gleaned from receivers have to be transmitted to a potentially distant central unit. This requires either communications over long distances or complex multi-hop routing protocols, which may not be applicable for energy constrained WSNs. Moreover, centralized implementations are prone to link failures and exhibits poor scalability. By contrast, distributed estimation methods achieve low energy consumption by neighbor-to-neighbor communications and exhibit both high robustness and excellent scalability. They are especially competitive, when the location estimate of the target has to be available at each receiver. In [28], a consensus based method was proposed for solving a distributed hypothesis testing problem. A distributed target tracking algorithm based on likelihood based consensus was proposed in [29], where the global likelihood function can be determined by reaching consensus accorss all local likelihood functions. The distributed particle filter (DPF) [30] associated with likelihood consensus is proposed for target tracking in [31]. However, using this DPF requires the exchange of large packets of particles between neighboring receivers, leading to an unaffordable communications overhead.

In this paper, we focus our attention on the passive localization problem of asynchronous receivers in wireless networks using TOA measurements. We consider the scenario of targets equipped with RFID tags, therefore the signals arriving from the transmitter and reflected by different targets can be separated. In practical vehicular environments, having non-line-ofsight (NLOS) propagation is unavoidable, which imposes TOA delay even when all nodes in the network are synchronized [32]. Therefore we consider a mixed LOS/NLOS environment. We propose an expectation maximization (EM) based algorithm [33], [34] for iteratively estimating the location of passive targets in the maximization step (M-step), while we consider the clock offsets and NLOS biases to be hidden variables in the expectation step (E-step). A straightforward application of the EM algorithm would result in nonlinear expressions for the M-step. In order to avoid the application of numerical methods, we propose beneficial approximations that result in a closed-form solution for the M-step.

Secondly, we propose a consensus-based scheme for distributing the centralized processing tasks amongst the receivers. Since the conditioned expectation of the log-likelihood function obtaining from the E-step is constituted by a sum of the receivers' local functions, we rely on the average consensus of these local functions. These functions would require sophisticated particle representation for a direct implementation. In this treatise, we propose an approximate solution for the specific consensus problem. In summary, the main contributions of this paper are as follows:

- We extend the quasi-synchronous network assumption of [26] and propose a novel EM-based passive localization technique for asynchronous receivers (EMpLaR) operating in a mixed time-variant LOS/NLOS environment based on TOA measurements.

- We invoke the classic Taylor expansion for approximating the conditioned expectation of the log-likelihood function in the E-step, so that the M-step can be formulated analytically.

- A beneficial distributed implementation of passive localization is conceived. Then a quadratic polynomial approximation is proposed for restricting the local conditioned expectation of the log-likelihood function. As a result, only the coefficients of this quadratic polynomial have to be transmitted for arriving at the average consensus of the local functions, which significantly reduces the communications overhead imposed by the distributed implementation of EMpLaR.

- The Bayesian Cramér-Rao bound (BCRB) of the passive target location estimates is derived in the presence of clock offsets for evaluating the performance of the proposed algorithms.

The remainder of this paper is organized as follows. In Section II, the system model is presented. The centralized EMpLaR algorithm is proposed in Section III. A distributed implementation based on the average consensus of the local functions is established in Section IV. The Bayesian CramérRao lower bound of passive localization is derived in Section V for our asynchronous network. In Section VI, our simulation results and discussions are presented. Finally, our conclusions are offered in Section VII.

Notation: $(\cdot)^{\mathrm{T}}$ and $(\cdot)^{-1}$ are the transpose and the inverse operator, respectively; $\|\cdot\|$ denotes the Euclidean norm; $\mathbb{E}$ is the expectation operator; $\mathbb{R}$ denotes a real-valued number space; $\nabla_{x}$ represents the differential operator with respect to $x ; \operatorname{diag}\{\mathbf{x}\}$ is a diagonal matrix with the main diagonal entries being the elements of $\mathbf{x} ; \mathbf{A} \succeq \mathbf{B}$ denotes that $\mathbf{A}-\mathbf{B}$ is positive semi-definite matrix.

\section{SYSTEM MODEL}

We consider a network of $T=|\mathcal{T}|$ moving passive targets, a single transmitter and $A=|\mathcal{A}|$ receivers, where $\mathcal{T}$ and $\mathcal{A}$ denote the set of targets and receivers, respectively. The moving targets are equipped with RFID tags and the reflected signals can be separated at the receivers [35].

The transmitter is located at $\mathbf{x}_{\text {tran }}=\left[a_{0}, b_{0}\right]^{\mathrm{T}}$, while the receivers at $\boldsymbol{\kappa}_{n}=\left[a_{n}, b_{n}\right]^{\mathrm{T}}, n \in \mathcal{A}$. The coordinates of target 
$i$ at time $k$ are denoted as $\mathbf{x}_{i}^{k}=\left[x_{i}^{k}, y_{i}^{k}\right]^{\mathrm{T}}$. Considering the clock offset, the local clock of receiver $n$ is modeled by

$$
c_{n}(t)=t+\theta_{n}(t),
$$

where $\theta_{n}(t)$ denotes the time varying clock offset of receiver $n$ and $t$ is the reference time.

\section{A. Time Evolution Model}

According to [21], the clock offset at different time slots can be modeled as a memoryless Markov process obeying,

$$
\theta_{n}^{k}=\theta_{n}^{k-1}+\varepsilon,
$$

where $\varepsilon$ is a Gaussian noise process with zero mean and variance $\sigma_{\varepsilon}^{2}$. The state transition function of the $i$ th target is formulated as

$$
\mathbf{x}_{i}^{k}=\mathbf{x}_{i}^{k-1}+\delta_{t} \mathbf{v}_{i}^{k-1}+\boldsymbol{\Delta}_{v},
$$

where $\mathbf{v}_{i}^{k-1}$ is the velocity at time $k-1 ; \delta_{t}$ is the time slot duration and $\boldsymbol{\Delta}_{v}$ is a Gaussian state transition noise, $\boldsymbol{\Delta}_{v} \sim$ $\mathcal{N}\left(\mathbf{0}, \boldsymbol{\Sigma}_{v}\right)$.

The state transition probabilities can be obtained based on (1) and (2), formulated as,

$$
\begin{aligned}
& p\left(\mathbf{x}_{i}^{k} \mid \mathbf{x}_{i}^{k-1}\right) \propto \exp \left(-\frac{1}{2}\left\|\mathbf{x}_{i}^{k}-\mathbf{x}_{i}^{k-1}-\delta_{t} \mathbf{v}_{i}^{k-1}\right\|_{\Sigma_{v}^{-1}}^{2}\right), \\
& p\left(\theta_{n}^{k} \mid \theta_{n}^{k-1}\right) \propto \exp \left(-\frac{\left(\theta_{n}^{k}-\theta_{n}^{k-1}\right)^{2}}{2 \sigma_{\varepsilon}^{2}}\right) .
\end{aligned}
$$

\section{B. Measurement Model}

The transmitter emits a signal at the known reference instant $t=t_{0}^{k}$. This signal is reflected by target $i$ at reference instant $t_{i}^{\prime k}=t_{0}^{k}+\left\|\mathbf{x}_{\text {tran }}-\mathbf{x}_{i}^{k}\right\| / v$, where $v$ is the signal propagation velocity. The reflected signal arrives at receiver $n$ at reference instant $t_{i, n}^{k}=t_{i}^{\prime k}+\left\|\mathbf{x}_{i}^{k}-\boldsymbol{\kappa}_{n}\right\| / v, n \in \mathcal{A}$. Due to the clock offset, the receiver records this time with respect to its local clock, given by

$$
\begin{aligned}
c\left(t_{i, n}^{k}\right) & =t_{i, n}^{k}+\theta_{n}^{k}+\phi_{n} \\
& =t_{0}^{k}+\frac{\left\|\mathbf{x}_{\text {tran }}-\mathbf{x}_{i}^{k}\right\|+\left\|\mathbf{x}_{i}^{k}-\boldsymbol{\kappa}_{n}\right\|}{v}+\theta_{n}^{k}+\phi_{i, n},
\end{aligned}
$$

where $\phi_{i, n}$ is the measurement noise, which is assumed to be Gaussian distributed. Without loss of generality, we assume in the following that the transmitter is located at the origin, i.e., $a_{0}=b_{0}=0$.

Moving the term $t_{0}^{k}$ in (5) to the left hand side and multiplying both sides by the signal propagation velocity $v$, the LOS range measurement at instant $k$ can be obtained as

$$
R_{i, n}^{k}=\left\|\mathbf{x}_{i}^{k}\right\|+\left\|\mathbf{x}_{i}^{k}-\boldsymbol{\kappa}^{n}\right\|+v \theta_{n}^{k}+\omega_{i, n},
$$

where $\omega_{i, n}$ is a zero-mean Gaussian random variable with variance $\sigma_{i, n}^{2}$. However, due to the NLOS propagation, the range of measurements obtained at some of the receivers is biased by a positive term, yielding

$$
R_{i, n}^{k}=\left\|\mathbf{x}_{i}^{k}\right\|+\left\|\mathbf{x}_{i}^{k}-\boldsymbol{\kappa}^{n}\right\|+v \theta_{n}^{k}+\beta_{i, n}^{k}+\omega_{i, n} .
$$

In (7), $\beta_{i, n}^{k}$ denotes the positive bias caused by NLOS propagation, which obeys the negative-exponential distribution of [16],

$$
p\left(\beta_{i, n}^{k}\right)=\lambda e^{-\lambda \beta_{i, n}^{k}}, \quad \beta_{i, n}^{k}>0,
$$

and $\lambda$ is the parameter rate. ${ }^{2}$

Using $\mathcal{C}^{\mathrm{LOS}}$ and $\mathcal{C}^{\mathrm{NLOS}}$ to denote the pair of sets containing the LOS and NLOS links, the local likelihood function with respect to $R_{i, n}^{k}$ may be written as

$$
\begin{aligned}
& p\left(R_{i, n}^{k} \mid \mathbf{x}_{i}^{k}, \theta_{n}^{k}, \beta_{i, n}^{k}\right) \propto \\
& \begin{cases}\exp \left(-\frac{\left(R_{i, n}^{k}-\left\|\mathbf{x}_{i}^{k}\right\|-\left\|\mathbf{x}_{i}^{k}-\boldsymbol{\kappa}_{n}\right\|-v \theta_{n}^{k}\right)^{2}}{2 \sigma_{i, n}^{2}}\right) & (i, n) \in \mathcal{C}^{\mathrm{LOS}} \\
\exp \left(-\frac{\left(R_{i, n}^{k}-\left\|\mathbf{x}_{i}^{k}\right\|-\left\|\mathbf{x}_{i}^{k}-\boldsymbol{\kappa}_{n}\right\|-\beta_{i, n}^{k}-v \theta_{n}^{k}\right)^{2}}{2 \sigma_{i, n}^{2}}\right) & (i, n) \in \mathcal{C}^{\mathrm{NLOS}} .\end{cases}
\end{aligned}
$$

For brevity, we use $\mathbf{x}^{k}=\left[\left[\mathbf{x}_{1}^{k}\right]^{\mathrm{T}}, \ldots,\left[\mathbf{x}_{T}^{k}\right]^{\mathrm{T}}\right]^{\mathrm{T}}, \mathbf{R}^{k}=$ $\left[R_{1,1}^{k}, \ldots, R_{1, A}^{k}, \ldots, R_{i, 1}^{k}, \ldots, R_{i, A}^{k}, \ldots, R_{T, 1}^{k} \ldots, R_{T, A}^{k}\right]^{\mathrm{T}}, \quad \boldsymbol{\beta}^{k}=$ $\left[\beta_{1,1}^{k}, \ldots, \beta_{1, a}^{k}, \ldots, \beta_{i, 1}^{k}, \ldots, \beta_{i, A}^{k}, \ldots, \beta_{T, 1}^{k} \ldots, \beta_{T, A}^{k}\right]^{\mathrm{T}}$ and $\boldsymbol{\theta}^{k}=$ $\left[\theta_{1}^{k}, \ldots, \theta_{A}^{k}\right]^{\mathrm{T}}$ to denote all the target locations, range measurements, clock offsets and TOA biases caused by NLOS propagation at instant $k$, respectively. ${ }^{3}$ Since the noise at the different receivers is uncorrelated, the global likelihood function at instant $k$ is

$$
\begin{aligned}
p\left(\mathbf{R}^{k} \mid \mathbf{x}^{k}, \boldsymbol{\theta}^{k}, \boldsymbol{\beta}^{k}\right) \propto & \prod_{i \in \mathcal{T}} \prod_{(i, n) \in \mathcal{C}^{\operatorname{LOS}}} p\left(R_{i, n}^{k} \mid \mathbf{x}_{i}^{k}, \theta_{n}^{k}\right) \\
& \prod_{(i, n) \in \mathcal{C}^{\text {NLOS }}} p\left(R_{i, n}^{k} \mid \mathbf{x}_{i}^{k}, \theta_{n}^{k}, \beta_{i, n}^{k}\right) .
\end{aligned}
$$

Following the Bayesian rules, the joint a posteriori distribution at time $k$ is given by

$p\left(\mathbf{x}^{k}, \boldsymbol{\theta}^{k}, \boldsymbol{\beta}^{k} \mid \mathbf{R}^{k}\right) \propto p\left(\mathbf{R}^{k} \mid \mathbf{x}^{k}, \boldsymbol{\theta}^{k}, \boldsymbol{\beta}^{k}\right) \cdot p\left(\mathbf{x}^{k}\right) \cdot p\left(\boldsymbol{\theta}^{k}\right) \cdot p\left(\boldsymbol{\beta}^{k}\right)$,

where $p\left(\mathbf{x}^{k}\right), p\left(\boldsymbol{\theta}^{k}\right)$ and $p\left(\boldsymbol{\beta}^{k}\right)$ are the a priori distributions at time $k$. Our goal is to determine the target locations based on the set of measurements $\mathbf{R}^{k}$. Due to the presence of clock offsets, the two-step method (TSE) proposed in [24] is not suitable here.

\section{Centralized Expectation Maximization METHOD FOR PASSIVE LOCALIZATION}

In this section we derive a pair of EMpLaR algorithms, which differ in the choice of approximation used for finding the following maximum a posteriori (MAP) estimate of the target locations at instant $k$

$$
\begin{aligned}
\hat{\mathbf{x}}^{k} & =\underset{\mathbf{x}^{k}}{\arg \max } p\left(\mathbf{x}^{k} \mid \mathbf{R}^{k}\right) \\
& =\underset{\mathbf{x}^{k}}{\arg \max } \iint p\left(\mathbf{x}^{k}, \boldsymbol{\theta}^{k}, \boldsymbol{\beta}^{k} \mid \mathbf{R}^{k}\right) \mathrm{d} \boldsymbol{\theta}^{k} \mathrm{~d} \boldsymbol{\beta}^{k} .
\end{aligned}
$$

${ }^{2}$ Several contributions in the literature have been proposed to identify whether a measurement is LOS or NLOS [36], [37]. For simplicity, we assume that the range measurement received by a receiver has been perfectly identified to be LOS or NLOS.

${ }^{3}$ In some conditions, the receiver may not acquire the propagation signals reflected by the targets. As a result the measurement $R_{i, n}^{k}$ is removed from the corresponding vector. 


$$
\begin{aligned}
& g_{i}^{(l)}\left(\mathbf{x}_{i}\right)=-\sum_{n \in \mathcal{A}} \frac{1}{2 \sigma_{i, n}^{2}}\left(\left\|\mathbf{x}_{i}\right\|^{2}+2\left\|\mathbf{x}_{i}\right\|\left\|\mathbf{x}_{i}-\boldsymbol{\kappa}_{n}\right\|+\left\|\mathbf{x}_{i}-\kappa_{n}\right\|^{2}+2\left(v m_{\theta_{n}}^{(l)}-R_{i, n}\right) \cdot\left(\left\|\mathbf{x}_{i}\right\|+\left\|\mathbf{x}_{i}-\boldsymbol{\kappa}_{n}\right\|\right)\right) \\
&-\sum_{(i, n) \in \mathcal{C}^{\mathrm{NLOS}}} \frac{m_{\beta_{i, n}}^{(l)} \cdot\left(\left\|\mathbf{x}_{i}\right\|+\left\|\mathbf{x}_{i}-\boldsymbol{\kappa}_{n}\right\|\right)}{\sigma_{i, n}^{2}} \\
& h_{i}\left(\mathbf{x}_{i}\right)=-\left(\mathbf{x}_{i}-\hat{\mathbf{x}}_{i}^{k-1}-\delta_{t} \hat{\mathbf{v}}_{i}^{k-1}\right)^{T} \boldsymbol{\Sigma}_{v}^{-1}\left(\mathbf{x}_{i}-\hat{\mathbf{x}}_{i}^{k-1}-\delta_{t} \hat{\mathbf{v}}_{i}^{k-1}\right) . \\
& m_{\beta_{i, n}^{(l)}}^{(l)}=R_{i, n}-\left\|\hat{\mathbf{x}}_{i}^{(l-1)}\right\|-\left\|\hat{\mathbf{x}}_{i}^{(l-1)}-\boldsymbol{\kappa}_{n}\right\|-v m_{\theta_{n}}^{(l-1)}-\sigma_{i, n}^{2} \lambda \\
& m_{\theta_{n}}^{(l)}=\left(\frac{1}{\sigma_{\theta_{n}^{2}}^{2}+\sigma_{\varepsilon}^{2}}+\sum_{i} \frac{1}{\sigma_{i, n}^{2}}\right)^{-1}\left(\frac{\mu_{\theta_{n}^{k-1}}}{\sigma_{\theta_{n}^{k-1}}^{2}+\sigma_{\varepsilon}^{2}}-\sum_{(i, n) \in \mathcal{C}^{\mathrm{NLOs}}} \frac{m_{\beta_{i, n}}^{(l-1)}}{v \sigma_{i, n}^{2}}+\sum_{n} \frac{R_{i, n}-\left\|\hat{\mathbf{x}}_{i}^{(l-1)}\right\|-\left\|\hat{\mathbf{x}}_{i}^{(l-1)}-\boldsymbol{\kappa}_{n}\right\|}{v \sigma_{i, n}^{2}}\right),
\end{aligned}
$$

As mentioned in Section II, in the joint a posteriori distribution (11), $\mathbf{x}^{k}, \boldsymbol{\theta}^{k}$ and $\boldsymbol{\beta}^{k}$ are unknowns. Direct marginalization and using the MAP estimator is intractable.

Therefore, we conceive the corresponding EM algorithm [33] for solving (12) iteratively. ${ }^{4}$ We consider $\mathbf{R}^{k}$ as the incomplete data set, and $\left\{\mathbf{R}^{k}, \boldsymbol{\theta}^{k}, \boldsymbol{\beta}^{k}\right\}$ as the complete data set associated with the hidden variable $\boldsymbol{\theta}^{k}$ and $\boldsymbol{\beta}^{k}$. At the $l$ th iteration, the E-step calculates the conditioned expectation of the complete-data log augmented density derived in [38]. Then, the estimates of the target locations can be obtained in the M-step by maximizing the expression obtained in the E-step.

\section{A. Expectation Step}

For ease of exposition, we consider the $k$ th time slot. The superscript $k$ is dropped, wherever there is no confusion. According to [38], in E-step, the conditional expectation of the complete-data log augmented density is calculated by

$$
\begin{aligned}
Q & \left(\mathbf{x}, \hat{\mathbf{x}}^{(l-1)}\right) \propto \int p\left(\boldsymbol{\theta}, \boldsymbol{\beta} \mid \mathbf{R}, \hat{\mathbf{x}}^{(l-1)}\right) \cdot \ln p(\mathbf{R}, \boldsymbol{\theta}, \boldsymbol{\beta}, \mathbf{x}) \mathrm{d} \boldsymbol{\theta} \mathrm{d} \boldsymbol{\beta} \\
= & \int p\left(\boldsymbol{\theta}, \boldsymbol{\beta} \mid \mathbf{R}, \hat{\mathbf{x}}^{(l-1)}\right) \cdot \ln p(\mathbf{R} \mid \mathbf{x}, \boldsymbol{\theta}, \boldsymbol{\beta}) \mathrm{d} \boldsymbol{\theta} \mathrm{d} \boldsymbol{\beta}+\ln p(\mathbf{x})+C \\
& +\int p\left(\boldsymbol{\theta}, \boldsymbol{\beta} \mid \mathbf{R}, \hat{\mathbf{x}}^{(l-1)}\right) \cdot \ln p(\boldsymbol{\theta}, \boldsymbol{\beta}) \mathrm{d} \boldsymbol{\theta} \mathrm{d} \boldsymbol{\beta}
\end{aligned}
$$

where $C$ is a constant parameter, $p\left(\boldsymbol{\theta}, \boldsymbol{\beta} \mid \mathbf{R}, \hat{\mathbf{x}}^{(l-1)}\right)$ is the $a$ posteriori distribution of clock offsets and biases conditioned on the previous estimates $\hat{\mathbf{x}}^{(l-1)}$ of target locations and $p(\mathbf{x})$ is considered as the a priori information of target locations at time $k$. Here $p(\mathbf{x})$ is expressed as

$$
p(\mathbf{x})=\prod_{i \in \mathcal{T}} \int p\left(\mathbf{x}_{i}^{k} \mid \mathbf{x}_{i}^{k-1}\right) \cdot p\left(\mathbf{x}_{i}^{k-1}\right) \mathrm{d} \mathbf{x}_{i}^{k-1},
$$

which is a Gaussian distribution with a mean of $\hat{\mathbf{x}}_{i}^{k-1}+\delta_{t} \mathbf{v}_{i}^{k-1}$ and a covariance matrix of $\boldsymbol{\Sigma}_{v}$. For simplicity, we assume that the target velocity does not change within a single time slot and use $\hat{\mathbf{v}}_{i}^{k-1}=\left(\hat{\mathbf{x}}_{i}^{k-1}-\hat{\mathbf{x}}_{i}^{k-2}\right) / \delta_{t}$ for representing $\mathbf{v}_{i}^{k-1}$ in $p(\mathbf{x})$.

${ }^{4}$ The EM algorithm is originally presented to obtain the maximum likelihood estimate. As shown in [38], the EM algorithm can also be used in MAP problem.
The conditional a posteriori distribution can be rewritten as $p\left(\boldsymbol{\theta}, \boldsymbol{\beta} \mid \mathbf{R}, \hat{\mathbf{x}}^{(l-1)}\right) \propto p\left(\mathbf{R} \mid \boldsymbol{\theta}, \boldsymbol{\beta}, \hat{\mathbf{x}}^{(l-1)}\right) \cdot p(\boldsymbol{\theta}) \cdot p(\boldsymbol{\beta})$,

where $p\left(\mathbf{R} \mid \boldsymbol{\theta}, \boldsymbol{\beta}, \hat{\mathbf{x}}^{(l-1)}\right)$ is the likelihood function (10) with $\mathbf{x}$ been replaced by $\hat{\mathbf{x}}^{(l-1)}$ and $p(\boldsymbol{\beta})=\prod_{(i, n) \in \mathcal{C}^{\mathrm{NLOS}}} p\left(\beta_{i, n}\right)$. The a priori distribution of the receivers' clock offsets can be obtained by $p(\boldsymbol{\theta})=\prod_{n \in \mathcal{A}} \int p\left(\theta_{n}^{k} \mid \theta_{n}^{k-1}\right) p\left(\theta_{n}^{k-1}\right) \mathrm{d} \theta_{n}^{k-1}$. Given the mean and covariance matrix of $\boldsymbol{\theta}^{k-1}$ denoted as $\boldsymbol{\mu}_{\boldsymbol{\theta}}^{k-1}=$ $\left[\mu_{\theta_{1}^{k-1}}, \ldots, \mu_{\theta_{A}^{k-1}}\right]^{\mathrm{T}}$ and $\mathbf{V}_{\boldsymbol{\theta}}^{k-1}=\operatorname{diag}\left\{\sigma_{\theta_{1}^{k-1}}^{2}, \ldots, \sigma_{\theta_{A}^{k-1}}^{2}\right\}$, according to (4), $p(\boldsymbol{\theta})$ is a Gaussian distribution with mean $\boldsymbol{\mu}_{\boldsymbol{\theta}}^{k-1}$ and covariance matrix $\mathbf{V}_{\boldsymbol{\theta}}^{k-1}+\sigma_{\varepsilon}^{2} \mathbf{I}$.

Dropping the terms that are irrelevant to $\mathbf{x}$, the conditioned expected log augmented density can be calculated as

$$
Q\left(\mathbf{x}, \hat{\mathbf{x}}^{(l-1)}\right)=\sum_{i \in \mathcal{T}} g_{i}^{(l)}\left(\mathbf{x}_{i}\right)+\sum_{i \in \mathcal{T}} h_{i}\left(\mathbf{x}_{i}\right),
$$

where $g_{i}^{(l)}\left(\mathbf{x}_{i}\right)$ and $h_{i}\left(\mathbf{x}_{i}\right)$ are defined in (16) and (17). In (16), $m_{\beta_{i, n}}^{(l)}$ and $m_{\theta_{i, n}}^{(l)}$ are defined in (18) and (19). Note that for each target, the conditioned expected log augmented density can be calculated separately. In what follows, we will consider the estimate of the $i$ th target's location.

\section{B. Maximization Step}

In the M-step, the target location estimate $\hat{\mathbf{x}}^{(l)}$ is obtained by maximizing $Q\left(\mathbf{x}, \hat{\mathbf{x}}^{(l-1)}\right)$, which is formulated as,

$$
\begin{aligned}
\hat{\mathbf{x}}^{(l)} & =\underset{\mathbf{x}}{\arg \max }\left[Q\left(\mathbf{x}, \hat{\mathbf{x}}^{(l-1)}\right)\right] \\
& =\underset{\mathbf{x}}{\arg \max }\left[\sum_{i}\left(g_{i}^{(l)}\left(\mathbf{x}_{i}\right)+h_{i}\left(\mathbf{x}_{i}\right)\right)\right],
\end{aligned}
$$

where $g_{i}^{(l)}\left(\mathbf{x}_{i}\right)$ and $h_{i}\left(\mathbf{x}_{i}\right)$ are defined in (16) and (17). Consequently we have the estimate of target $i$ 's location as

$$
\hat{\mathbf{x}}_{i}^{(l)}=\arg \max _{\mathbf{x}_{i}}\left[g_{i}^{(l)}\left(\mathbf{x}_{i}\right)+h_{i}\left(\mathbf{x}_{i}\right)\right] .
$$

Let us set the derivative of $g_{i}^{(l)}\left(\mathbf{x}_{i}\right)+h_{i}\left(\mathbf{x}_{i}\right)$ with respect to $\mathbf{x}_{i}$ to zero, yielding,

$$
\frac{\partial\left[g_{i}^{(l)}\left(\mathbf{x}_{i}\right)+h_{i}\left(\mathbf{x}_{i}\right)\right]}{\partial \mathbf{x}_{i}}=0 .
$$


Note that in EM iterations, the term $\partial h_{i}\left(\mathbf{x}_{i}\right) / \partial \mathbf{x}_{i}=$ $\left[\frac{x_{i}-\hat{x}_{i}^{k-1}-\delta_{t} \hat{v}_{x_{i}}}{\sigma_{v_{x}}^{2}}, \frac{y_{i}-\hat{y}_{i}^{k-1}-\delta_{t} \hat{v}_{y_{i}}}{\sigma_{v_{y}}^{2}}\right]^{\mathrm{T}}$ remains unchanged. Nonetheless, due to the terms $\left\|\mathbf{x}_{i}\right\|$ and $\left\|\mathbf{x}_{i}-\boldsymbol{\kappa}_{n}\right\|$ in $g_{i}^{(l)}\left(\mathbf{x}_{i}\right)$, we still can not directly determine the target location from (22). Since the relevant numerical methods suffer from a high computational burden, we invoke the classic Taylor expansion for approximating the nonlinear terms in (15) such that the estimate $\hat{\mathbf{x}}_{i}^{(l)}$ can be determined in closed form.

Explicitly, we approximate the terms $\left\|\mathbf{x}_{i}\right\|,\left\|\mathbf{x}_{i}-\boldsymbol{\kappa}_{n}\right\|$ and $\left\|\mathbf{x}_{i}\right\| \cdot\left\|\mathbf{x}_{i}-\boldsymbol{\kappa}_{n}\right\|$ in (15) by the first- and the second-order Taylor series expansions around the previous estimate $\hat{\mathbf{x}}_{i}^{(l-1)}$. For both approximations, (22) can be rewritten as

$$
\left\{\begin{array}{c}
A_{i}^{(l-1)} \hat{x}_{i}^{(l)}+B_{i}^{(l-1)} \hat{y}_{i}^{(l)}=D_{i}^{(l-1)}, \\
B_{i}^{(l-1)} \hat{x}_{i}^{(l)}+C_{i}^{(l-1)} \hat{y}_{i}^{(l)}=E_{i}^{(l-1)},
\end{array}\right.
$$

where the coefficients in the expressions are derived in Appendix A. The solution of (23), i.e., $\hat{\mathbf{x}}_{i}^{(l)}=\left[\hat{x}_{i}^{(l)}, \hat{y}_{i}^{(l)}\right]^{\mathrm{T}}$, is given by

$$
\begin{aligned}
& \hat{x}_{i}^{(l)}=\frac{D_{i}^{(l-1)} C_{i}^{(l-1)}-E_{i}^{(l-1)} B_{i}^{(l-1)}}{A_{i}^{(l-1)} C_{i}^{(l-1)}-\left(B_{i}^{(l-1)}\right)^{2}}, \\
& \hat{y}_{i}^{(l)}=\frac{E_{i}^{(l-1)} A_{i}^{(l-1)}-D_{i}^{(l-1)} B_{i}^{(l-1)}}{A_{i}^{(l-1)} C_{i}^{(l-1)}-\left(B_{i}^{(l-1)}\right)^{2}} .
\end{aligned}
$$

The coefficients are chosen differently for the first- and second-order Taylor expansion and will affect the localization accuracy and the complexity. The analysis will be given in the simulation results.

The proposed centralized EMpLaR algorithms based on the first- and second-order Taylor expansions are summarized in Algorithm 1. They differ in the choice of the parameters in (24) and (25) in line 7, explicitly for the first-order Taylor series expansion the set (47)-(50) is used, while for the second-order Taylor expansion the set (52)-(56) is employed.

\section{Distributed Implementation Based on Average CONSENSUS}

The previously proposed EMpLaR methods and most of the existing contributions on passive localization assume the existence of a fusion center. Transmitting the data of each receiver to a potentially distant central unit results in high energy cost in large-scale WSNs. Moreover, the centralized method is prone to link failure and to the malfunctioning of the fusion center. By contrast, distributed estimation techniques using only local processing and local communications with the neighboring receivers are highly robust and eminently scalable. In this section, we will first review the likelihood consensus based distributed estimation and then propose an average consensus based method for distributed passive localization in asynchronous networks.

\section{A. Likelihood Consensus}

Observe from (10) that the likelihood function corresponding to target $i$ at a specific time slot is the product of several local likelihood functions obtained at the receivers.

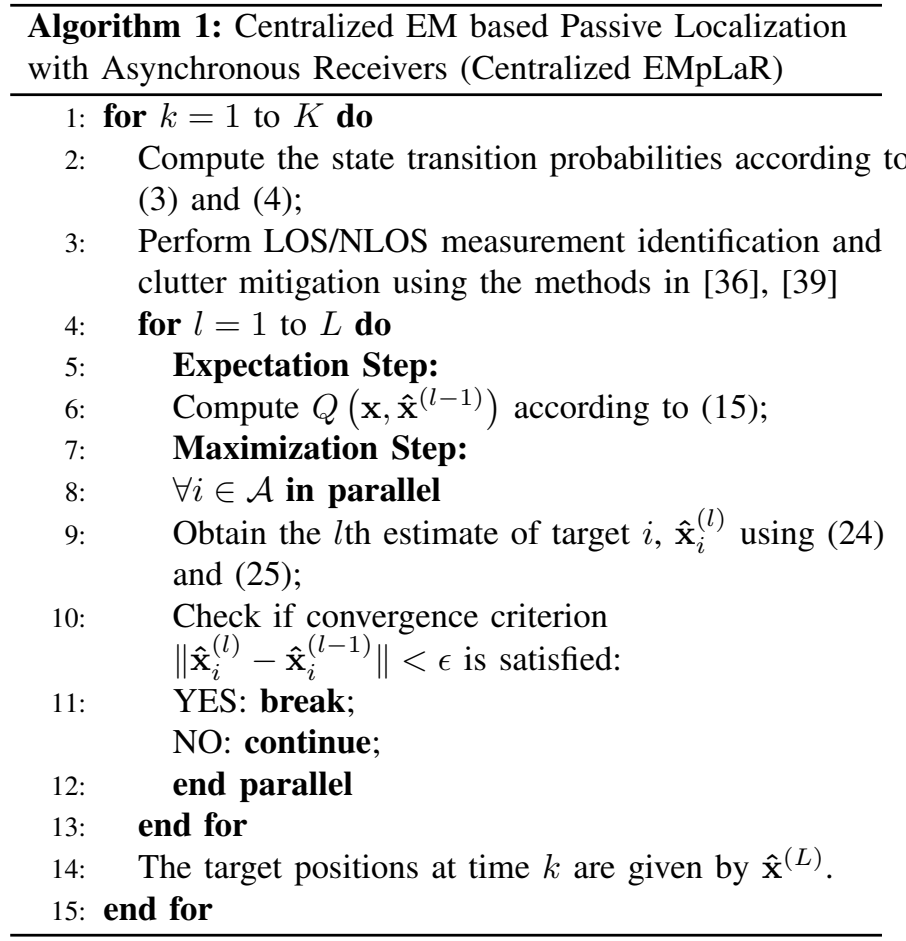

The likelihood consensus and DPF method aim for obtaining $p\left(\mathbf{R}_{i} \mid \mathbf{x}_{i}, \boldsymbol{\theta}, \boldsymbol{\beta}\right)$ in a distributed way by exchanging particles and the corresponding weights amongst the neighboring receivers.

In the presence of clock offsets and NLOS biases, the particles should be sampled on an extended space i.e., $\mathbf{q}_{i}=$ $\left[\mathbf{x}_{i}^{\mathrm{T}}, \boldsymbol{\theta}^{\mathrm{T}}, \boldsymbol{\beta}^{\mathrm{T}}\right]^{\mathrm{T}}$, and the local likelihood function can be represented by $J$ particles $\left\{\mathbf{q}_{i}^{(j)}, \varrho_{i, n}^{(j)}\right\}_{j=1}^{J}$ as

$$
p\left(R_{i, n} \mid \mathbf{x}_{i}, \theta_{n}, \beta_{i, n}\right) \simeq \sum_{j=1}^{J} \varrho_{i, n}^{(j)} p\left(R_{i, n} \mid \mathbf{q}_{i}^{(j)}\right) .
$$

Then the likelihood consensus is performed by exchanging and updating the local weights of the particles in a canonical form. After a few iterations, each receiver obtains a good approximation of the global likelihood function represented by its local particles.

Nonetheless, using a particle-based representation of the local function means that a large number of particles and their corresponding weights have to be exchanged amongst the neighboring receivers, which is rather wasteful both in terms of power and bandwidth.

\section{B. The Proposed Average Consensus-based Passive Localiza- tion}

Let us now adapt ideas from the likelihood consensus method of Sec. IV-A and propose an average consensus based scheme for obtaining the target location locally at each receiver.

Based on (16), we can observe that $g_{i}^{(l)}\left(\mathbf{x}_{i}\right)$ is the sum of the local log-likelihood functions over the conditioned 
expectation, i.e.,

$$
g_{i}^{(l)}\left(\mathbf{x}_{i}\right)=\sum_{n} g_{i, n}^{(l)}\left(\mathbf{x}_{i}\right)
$$

where $g_{i, n}^{(l)}\left(\mathbf{x}_{i}\right)$ is expressed in (27).

$$
g_{i, n}^{(l)}\left(\mathbf{x}_{i}\right) \triangleq\left\{\begin{array}{c}
-\frac{1}{2 \sigma_{i, n}^{2}}\left(\left\|\mathbf{x}_{i}\right\|^{2}+2\left\|\mathbf{x}_{i}\right\|\left\|\mathbf{x}_{i}-\boldsymbol{\kappa}_{n}\right\|+\left\|\mathbf{x}_{i}-\boldsymbol{\kappa}_{n}\right\|^{2}\right. \\
\left.+2\left(v m_{\theta_{n}}^{(l)}-R_{i, n}\right) \cdot\left(\left\|\mathbf{x}_{i}\right\|+\left\|\mathbf{x}_{i}-\boldsymbol{\kappa}_{n}\right\|\right)\right) \\
(i, n) \in \mathcal{C}^{\mathrm{LOS}} \\
-\frac{1}{2 \sigma_{i, n}^{2}}\left(\left\|\mathbf{x}_{i}\right\|^{2}+2\left\|\mathbf{x}_{i}\right\|\left\|\mathbf{x}_{i}-\boldsymbol{\kappa}_{n}\right\|+\left\|\mathbf{x}_{i}-\boldsymbol{\kappa}_{n}\right\|^{2}\right. \\
\left.+2\left(m_{\beta_{i, n}}^{(l)}+v m_{\theta_{n}}^{(l)}-R_{i, n}\right) \cdot\left(\left\|\mathbf{x}_{i}\right\|+\left\|\mathbf{x}_{i}-\boldsymbol{\kappa}_{n}\right\|\right)\right) \\
(i, n) \in \mathcal{C}^{\mathrm{NLOS}} .
\end{array}\right.
$$

Again, our goal is to obtain $g_{i}^{(l)}\left(\mathbf{x}_{i}\right)$ by exchanging parameters of $g_{i, n}^{(l)}\left(\mathbf{x}_{i}\right), n \in \mathcal{A}$, only amongst the neighboring receivers. $^{5}$

Denoting the iteration index of consensus processing by $p$, the standard average consensus is formulated as

$$
\begin{aligned}
& g_{i, n}^{(l),(p)}\left(\mathbf{x}_{i}\right)= \\
& g_{i, n}^{(l),(p-1)}\left(\mathbf{x}_{i}\right)+\xi \sum_{j \in \mathcal{N}_{n}}\left(g_{i, j}^{(l),(p-1)}\left(\mathbf{x}_{i}\right)-g_{i, n}^{(l),(p-1)}\left(\mathbf{x}_{i}\right)\right),
\end{aligned}
$$

where $\xi$ is the update rate and $\mathcal{N}_{n}$ is the set of all neighboring receivers of $n$.

In the standard average consensus method, all local receivers share the same update rate $\xi$, which may not provide good estimation performance. To tackle this problem, a so-called metropolis weight was defined in [40]. For the proposed EMpLaR scenario, this translates to the local update at receiver $n$, formulated as

$$
\begin{aligned}
g_{i, n}^{(l),(p)}\left(\mathbf{x}_{i}\right)=\xi_{n, n} g_{i, n}^{(l),(p-1)}\left(\mathbf{x}_{i}\right) & \\
& +\sum_{m \in \mathcal{N}_{n}} \xi_{m, n} g_{i, m}^{(l),(p-1)}\left(\mathbf{x}_{i}\right),
\end{aligned}
$$

with the metropolis update rate expressed as

$$
\xi_{n, m}=\xi_{m, n}=\left\{\begin{array}{cl}
1 / \max \left(\left|\mathcal{N}_{n}\right|,\left|\mathcal{N}_{m}\right|\right), & \text { for } n \neq m \\
1-\sum_{m^{\prime} \in \mathcal{N}_{n}} \xi_{m^{\prime}, n}, & \text { for } n=m .
\end{array}\right.
$$

where $\left|\mathcal{N}_{n}\right|$ and $\left|\mathcal{N}_{m}\right|$ denote the number of receivers next to receiver $n$ and $m$, respectively. Since the positions of the transmitter and receivers are known, the metropolis rate can be obtained before performing passive localization.

After $N_{c}$ iterations, all receivers reach consensus on the local functions, which are expressed as, $g_{i, n}^{(l),\left(N_{c}\right)}\left(\mathbf{x}_{i}\right) \simeq$ $g_{i, m}^{(l),\left(N_{c}\right)}\left(\mathbf{x}_{i}\right), n, m \in \mathcal{A}$ with $n \neq m$. Hence, the global function can be locally computed by

$$
g_{i}^{(l)}\left(\mathbf{x}_{i}\right)=A g_{i, n}^{(l),\left(N_{c}\right)}\left(\mathbf{x}_{i}\right) .
$$

The convergence of average consensus is guaranteed for a connected network [28].

${ }^{5}$ Note that $g_{i, n}^{(l)}\left(\mathbf{x}_{i}\right)$ varies in different EM iterations, therefore the average consensus process needs to be done after every E-step. Nonetheless, as shown in Section VI, the communications overhead of the proposed algorithm is still lower than that of the DPF based likelihood consensus.
In the consensus process, the local functions $g_{i, n}^{(l)}\left(\mathbf{x}_{i}\right)$, $n \in \mathcal{A}$, which have to be exchanged amongst the neighboring receivers through wireless channels are continuous functions of the variable $\mathbf{x}_{i}$. A straightforward approximation is to use the particle representation of $\mathbf{x}_{i}$ by $\left\{\mathbf{x}_{i}^{(j)}, \varrho_{i}^{(j)}\right\}$. However, the estimation accuracy is restricted by the number of particles employed. To achieve accurate localization, large packets containing a high number of particles have to be exchanged amongst the neighboring receivers.

For the sake of having a low communications overhead, we approximate the local function $g_{i, n}^{(l)}\left(\mathbf{x}_{i}\right)$ in quadratic polynomial form. Using the first-order Taylor expansion around the previous estimate $\left[\hat{x}_{i}^{(l-1)}, \hat{y}_{i}^{(l-1)}\right]^{\mathrm{T}},(27)$ can be approximated by

$$
g_{i, n}^{(l)}\left(\mathbf{x}_{i}\right) \approx-\frac{\left(x_{i}-\bar{u}_{i, n}^{(l)}\right)^{2}+\left(y_{i}-\bar{w}_{i, n}^{(l)}\right)^{2}}{\Sigma_{i, n}^{(l)}},
$$

where $\Sigma_{i, n}^{(l)}=\sigma_{i, n}^{2}$ and $\bar{u}_{i, n}^{(l)}$ and $\bar{w}_{i, n}^{(l)}$ are given as

$$
\begin{gathered}
\bar{u}_{i, n}^{(l)}=\frac{a_{n}}{2}-\frac{\left(\hat{d}_{i, n}^{(l-1)}\right)^{2} \hat{x}_{i}^{(l-1)}+\left(\hat{d}_{i}^{(l-1)}\right)^{2}\left(\hat{x}_{i}^{(l-1)}-a_{n}\right)}{2 \hat{d}_{i}^{(l-1)} \hat{d}_{i, n}^{(l-1)}} \\
\left.+\frac{R_{i, n}-v m_{\theta_{n}}^{(l)}-m_{\beta_{i, n}}^{(l)}\left(\frac{\hat{x}_{i}^{(l-1)}}{2}+\frac{\hat{x}_{i}^{(l-1)}-a_{n}}{\hat{d}_{i}^{(l-1)}}\right),}{\hat{d}_{i, n}^{(l-1)}}\right) \\
\bar{w}_{i, n}^{(l)}=\frac{b_{n}}{2}-\frac{\left(\hat{d}_{i, n}^{(l-1)}\right)^{2} \hat{y}_{i}^{(l-1)}+\left(\hat{d}_{i}^{(l-1)}\right)^{2}\left(\hat{y}_{i}^{(l-1)}-b_{n}\right)}{2 \hat{d}_{i}^{(l-1)} \hat{d}_{i, n}^{(l-1)}} \\
+\frac{R_{i, n}-v m_{\theta_{n}}^{(l)}-m_{\beta_{i, n}}^{(l)}}{2}\left(\frac{\hat{y}_{i}^{(l-1)}}{\hat{d}_{i}^{(l-1)}}+\frac{\hat{y}_{i}^{(l-1)}-b_{n}}{\hat{d}_{i, n}^{(l-1)}}\right),
\end{gathered}
$$

with $\hat{d}_{i}^{(l-1)} \triangleq\left\|\hat{\mathbf{x}}_{i}^{(l-1)}\right\|$ and $\hat{d}_{i, n}^{(l-1)} \triangleq\left\|\boldsymbol{\kappa}_{n}-\hat{\mathbf{x}}_{i}^{(l-1)}\right\|$. For the LOS link, we set $m_{\beta_{i, n}}^{(l)}=0$.

Building on the quadratic polynomial approximation, in the consensus process, only the parameters $\bar{u}_{i, n}, \bar{w}_{i, n}$ and $\Sigma_{i, n}$ have to be exchanged amongst the receivers, which significantly reduces the communications overhead.

Given the metropolis update rate, the local function at the $p$ th consensus iteration can be expressed as

$$
g_{i, n}^{(l),(p)}\left(\mathbf{x}_{i}\right)=-\frac{\left(x_{i}-\bar{u}_{i, n}^{(l),(p)}\right)^{2}+\left(y_{i}-\bar{w}_{i, n}^{(l),(p)}\right)^{2}}{\Sigma_{i, n}^{(l),(p)}},
$$

with the parameters $\bar{u}_{i, n}^{(l),(p)}, \bar{w}_{i, n}^{(l),(p)}$ and $\Sigma_{i, n}^{(l),(p)}$ updated as

$$
\begin{aligned}
& \Sigma_{i, n}^{(l),(p)}=\left(\frac{\xi_{n, n}}{\Sigma_{i, n}^{(l),(p-1)}}+\sum_{m \in \mathcal{N}_{n}} \frac{\xi_{n, m}}{\Sigma_{i, m}^{(l),(p-1)}}\right)^{-1}, \\
& \bar{u}_{i, n}^{(l),(p)}=\Sigma_{i, n}^{(l),(p)}\left(\frac{\xi_{n, n} \bar{u}_{i, n}^{(l),(p-1)}}{\Sigma_{i, n}^{(l),(p-1)}}+\sum_{m \in \mathcal{N}_{n}} \frac{\xi_{n, m} \bar{u}_{u, m}^{(l),(p-1)}}{\Sigma_{i, m}^{(l),(p-1)}}\right),
\end{aligned}
$$

$$
\bar{w}_{i, n}^{(l),(p)}=\Sigma_{i, n}^{(l),(p)}\left(\frac{\xi_{n, n} \bar{w}_{i, n}^{(l),(p-1)}}{\Sigma_{i, n}^{(l),(p-1)}}+\sum_{m \in \mathcal{N}_{n}} \frac{\xi_{n, m} \bar{w}_{i, m}^{(l),(p-1)}}{\Sigma_{i, m}^{(l),(p-1)}}\right) .
$$


At the $l$ th EM iteration, the local functions $g_{i, n}^{(l)}\left(\mathbf{x}_{i}\right)$ are initialized according to (31) with the parameters (32) and (33), i.e., $\bar{u}_{i, n}^{(l),(0)}=\bar{u}_{i, n}^{(l-1),\left(N_{c}\right)}$ and $\bar{w}_{i, n}^{(l),(0)}=\bar{w}_{i, n}^{(l),\left(N_{c}\right)}$. After convergence, every receiver obtains the global function $g_{i}^{(l)}\left(\mathbf{x}_{i}\right)$ and becomes capable of maximizing it to obtain $\hat{\mathbf{x}}_{i}^{(l)}$ individually. By observing (22), (30) and (31), solution of the M-step at the $l$ th EM iteration is

$$
\begin{aligned}
& \hat{x}_{i}^{(l)}=\frac{\sigma_{v_{x}}^{2} \bar{u}_{i, n}^{(l),\left(N_{c}\right)}+\Sigma_{i, n}^{(l),\left(N_{c}\right)}\left(\hat{x}_{i}^{(l-1)}+\delta_{t} \hat{v}_{x_{i}}^{k-1}\right)}{\Sigma_{i, n}^{(l),\left(N_{c}\right)}+\sigma_{v_{x}}^{2}}, \\
& \hat{y}_{i}^{(l)}=\frac{\sigma_{v_{y}}^{2} \bar{w}_{i, n}^{(l),\left(N_{c}\right)}+\Sigma_{i, n}^{(l),\left(N_{c}\right)}\left(\hat{y}_{i}^{(l-1)}+\delta_{t} \hat{v}_{y_{i}}^{k-1}\right)}{\Sigma_{i, n}^{(l),\left(N_{c}\right)}+\sigma_{v_{y}}^{2}} .
\end{aligned}
$$

The proposed distributed EMpLaR algorithm based on the average consensus of the local functions is summarized in Algorithm 2.

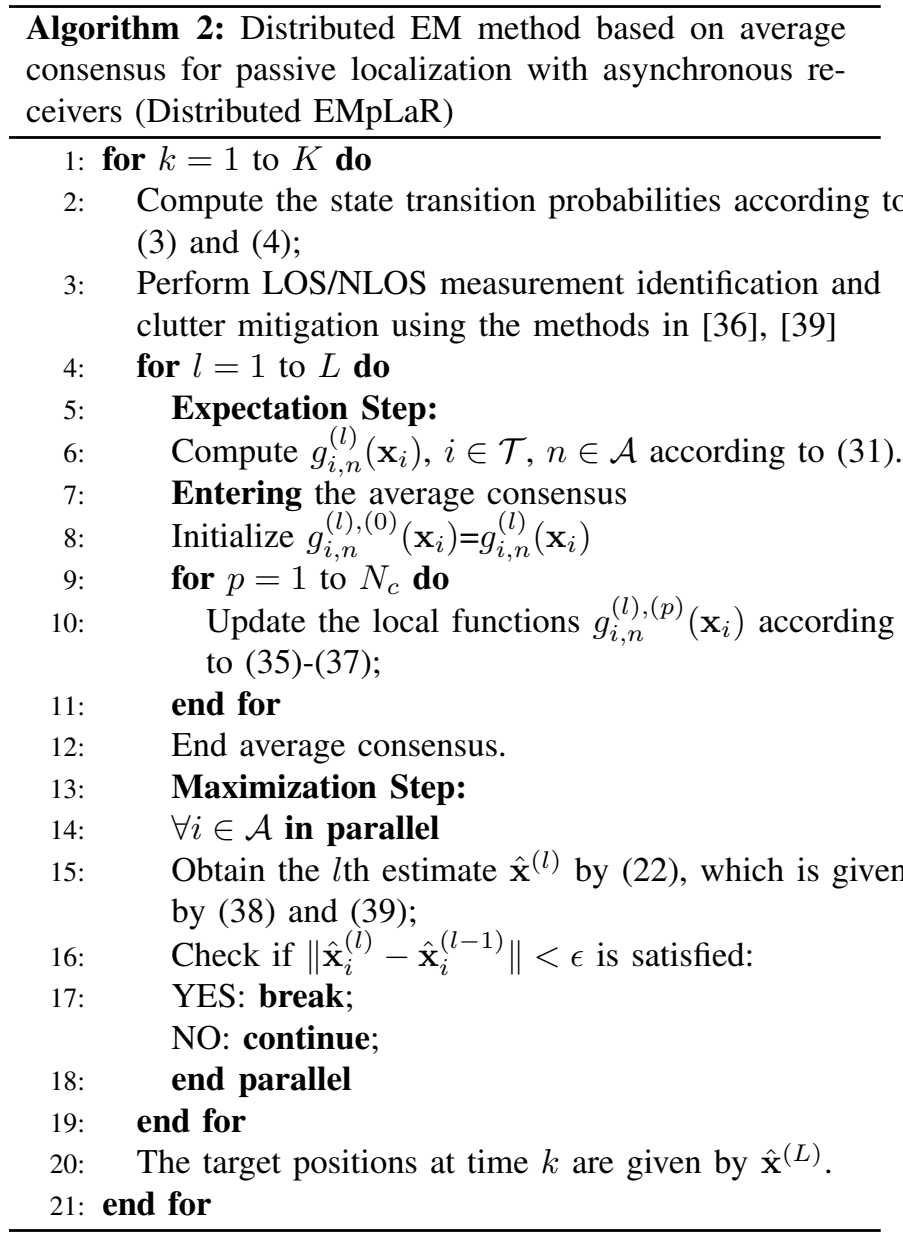

\section{CRAMÉR-RAO BOUND}

The Cramér-Rao Bound is a lower bound on the variance of unbiased estimators, which provides a benchmark for evaluating the performance of the proposed EMpLaR algorithms. Considering the CRB at time slot $k$, let $\boldsymbol{\tau} \triangleq[\mathbf{x}, \boldsymbol{\theta}, \boldsymbol{\beta}]^{T}$. The Bayesian CRB for unknown variable estimates is given by $\operatorname{Cov}(\hat{\boldsymbol{\tau}}) \succeq \mathbf{F}^{-1}$, where $\mathbf{F}$ is the Fisher information matrix (FIM) defined in,
$\mathbf{F}=\underbrace{-\mathbb{E}\left[\nabla_{\boldsymbol{\tau}}\left\{\nabla_{\boldsymbol{\tau}}\left(\ln p\left(\mathbf{R}^{k} \mid \boldsymbol{\tau}\right)\right)\right\}\right]}_{\mathbf{F}_{1}} \underbrace{-\mathbb{E}\left[\nabla_{\boldsymbol{\tau}}\left\{\nabla_{\boldsymbol{\tau}}(\ln p(\boldsymbol{\tau}))\right\}\right]}_{\mathbf{F}_{2}}$.

where $\mathbf{F}_{1}$ can be derived as

$$
\mathbf{F}_{1}=\mathbf{J} \boldsymbol{\Sigma}^{-1} \mathbf{J}^{\mathrm{T}}
$$

with $\boldsymbol{\Sigma} \in \mathbb{R}^{T A \times T A}$ being a diagonal matrix, obeying

$$
\boldsymbol{\Sigma}=\operatorname{diag}\left\{\sigma_{11}^{2}, \ldots, \sigma_{T A}^{2}\right\},
$$

and $\mathbf{J}$ is an $(A+2 T+T A) \times T A$-element Jacobian matrix with respect to $\tau$ expressed as

$$
\mathbf{J}=\left[\begin{array}{c}
\mathbf{P} \\
v \mathbf{I}_{A} \\
\mathbf{B}
\end{array}\right]
$$

$\mathbf{I}_{A} \in \mathbb{R}^{A \times A}$ is an identity matrix and $\mathbf{P} \in \mathbb{R}^{2 T \times T A}$ is a Jacobian matrix with respect to $\mathbf{x}$ having the elements of

$$
\begin{array}{r}
\mathbf{P}_{2 i-1, i n}=\frac{x_{i}-a_{n}}{\sqrt{\left(x_{i}-a_{n}\right)^{2}+\left(y_{i}-b_{n}\right)^{2}}}+\frac{x_{i}}{\sqrt{x_{i}^{2}+y_{i}^{2}}}, \\
\mathbf{P}_{2 i, i n}=\frac{y_{i}-b_{n}}{\sqrt{\left(x_{i}-a_{n}\right)^{2}+\left(y_{i}-b_{n}\right)^{2}}}+\frac{y_{i}}{\sqrt{x_{i}^{2}+y_{i}^{2}}} i \\
1 \leq i \leq T \text { and } 1 \leq n \leq A,
\end{array}
$$

where $\mathbf{B}$ can be regarded as an adjacency matrix, i.e., $B_{i, n}=1$ if and only if $\beta_{i, n}^{k} \neq 0$.

Furthermore, $\mathbf{F}_{2} \in \mathbb{R}^{(2 T+A+T A) \times(2 T+A+T A)}$ of (40) is related to the state transition functions, formulated as

$$
\mathbf{F}_{2}=\left[\begin{array}{cc}
\mathbf{I}_{A} \otimes \boldsymbol{\Sigma}_{v}^{-1} & \mathbf{0} \\
\mathbf{0} & \left(\mathbf{V}_{\boldsymbol{\theta}^{k-1}}+\sigma_{\varepsilon}^{2} \mathbf{I}_{R}\right)^{-1}
\end{array}\right]
$$

where $\otimes$ denotes the Kronecker product.

Given $\mathbf{F}_{1}$ and $\mathbf{F}_{2}$ of (40), $\mathbf{F}$ can partitioned as

$$
\mathbf{F}=\left[\begin{array}{ll}
\mathbf{F}_{11} & \mathbf{F}_{12} \\
\mathbf{F}_{12}^{\mathrm{T}} & \mathbf{F}_{22}
\end{array}\right]
$$

where we have $\mathbf{F}_{11} \in \mathbb{R}^{2 T \times 2 T}, \mathbf{F}_{12} \in \mathbb{R}^{2 T \times(A+T A)}, \mathbf{F}_{22} \in$ $\mathbb{R}^{(A+T A) \times(A+T A)}$

Then, the mean squared error (MSE) of the target location estimates at instant $k$ is bounded by

$$
\operatorname{MSE}\left(\hat{\mathbf{x}}^{k}\right) \geq \operatorname{Tr}\left(\left[\mathbf{F}^{-1}\right]_{\mathbf{x}^{k}}\right)
$$

where $\left[\mathbf{F}^{-1}\right]_{\mathbf{x}^{k}}$ denotes the square submatrix on the diagonal of $\mathbf{F}^{-1}$ corresponding to $\mathbf{x}^{k}$. However, calculating (44) directly requires the inversion of $\mathbf{F}$. Hence, the computational complexity increases significantly as the number of receivers $R$ becomes larger. Moreover, the condition number of the matrix $\mathbf{F}$ is usually high, which may lead to inaccurate results in numerical computing. To this end, we use the equivalent Fisher information matrix (EFIM) defined in [41] instead of the FIM. Explicitly, the EFIM of $\mathbf{x}^{k}$ denoted as $\mathbf{F}_{\mathbf{x}^{k}}$ satisfies the equality $\left[\mathbf{F}_{\mathbf{x}^{k}}\right]^{-1}=\left[\mathbf{F}^{-1}\right]_{\mathbf{x}^{k}}$, which means that all the information related to target has been retained. As shown in [41], the EFIM $\mathbf{F}_{\mathbf{x}^{k}}$ is the Schur complement of the matrix $\mathbf{F}_{22}$ in $\mathbf{F}$, which is expressed as

$$
\mathbf{F}_{\mathbf{x}^{k}}=\mathbf{F}_{11}-\mathbf{F}_{12} \mathbf{F}_{22}^{-1} \mathbf{F}_{12}^{T} .
$$


Calculating the inverse of $\mathbf{F}_{\mathbf{x}^{k}}$ has much a lower complexity than that of F. Similarly, the CRB of the clock offset can also be determined.

\section{Vi. Simulation Results And Discussions}

In this section, we will evaluate the performance of the proposed centralized and distributed EMpLaR algorithms. The performance inaccuracies due to the first- and the second-order Taylor expansions are considered first. Then, the impact of receiver configurations and the number of consensus iterations are investigated. Finally, we compare the proposed EMpLaR algorithms to state-of-the-art methods in terms of their estimation accuracy versus both their computational complexity and communications overhead.

\section{A. Simulation Setup}

TABLE I

SIMULATION PARAMETERS

\begin{tabular}{|c|c|}
\hline Parameter & Value \\
\hline Receiver positions & $\begin{array}{r}\mathbf{x}_{1}=[20,10]^{\mathrm{T}}, \mathbf{x}_{2}=[40,25]^{\mathrm{T}}, \mathbf{x}_{3}=[70,70]^{\mathrm{T}}, \\
\mathbf{x}_{4}=[25,40]^{\mathrm{T}}, \mathbf{x}_{5}=[10,20]^{\mathrm{T}}\end{array}$ \\
\hline Clock bias & $\mu_{\theta_{n}}=1 \mu \mathrm{s}, \sigma_{\theta_{n}}=100 \mathrm{~ns}^{6}$ \\
\hline Measurement noise & $\sigma_{i n n}^{2}=1 m^{2}$ \\
\hline Time interval & $\Delta_{t}=1 \mathrm{~s}$ \\
\hline Communication range & $40 m$ \\
\hline State transition noise & $\boldsymbol{\Sigma}_{v}=\operatorname{diag}\left\{1 m^{2}, 1 m^{2}\right\}, \sigma_{\varepsilon}=10 \mathrm{~ns}$ \\
\hline
\end{tabular}

Let us first consider the 2D localization of a passive target node on a $100 \times 100 \mathrm{~m}^{2}$ plane. The transmitter is always located at the origin, i.e. $\mathbf{x}_{\text {tran }}=[0,0]^{\mathrm{T}}$. Three targets are moving on the plane, whose velocity versus the $x$ and $y$-axis is uniformly generated from the range of $[0,3] \mathrm{m} / \mathrm{s}$. The initial locations of the targets are randomly distributed on this plane, while the guess of their locations is chosen to be the center of the 2D plane, i.e. $\hat{\mathbf{x}}_{i}^{0,(0)}=[50,50]^{\mathrm{T}}$. For simplicity, we assume that the measurement at a receiver with respect to a target is either related to a LOS link or NLOS link. The percentage of NLOS links is $10 \%$ and the bias obeys the exponential distribution of equation (8) having the parameter of $\lambda=0.35 m^{-1}$. The maximum number of EM iterations $L$ and the maximum number of consensus iterations $N_{c}$ are set to $L=N_{c}=50$.

\section{B. Performance of the Proposed EMpLaR}

We first analyze the root mean squared error (RMSE) of the estimated target location versus the number of EM iterations. In Fig. 1 the RMSE of target locations determined by the centralized EMpLaR algorithm at the time slot $k=10$ using the first- and the second-order Taylor expansions is depicted. The performance of our numerical method is also shown as a reference. It can be observed that both Taylor expansions converge to the RMSE of the numerical method, while the second-order approximation is converging faster at the cost of a higher computational complexity. This enables us to strike a compromise between the estimation accuracy versus the complexity in practical applications. In what follows, the

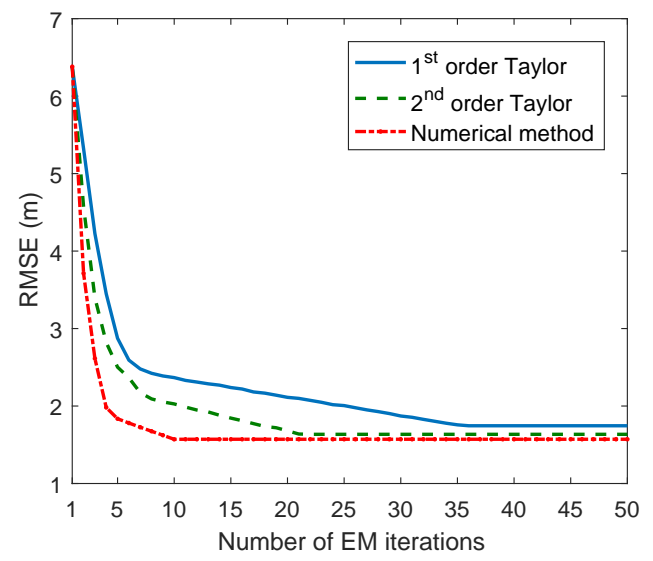

Fig. 1. RMSE of target location versus the number of EM iterations

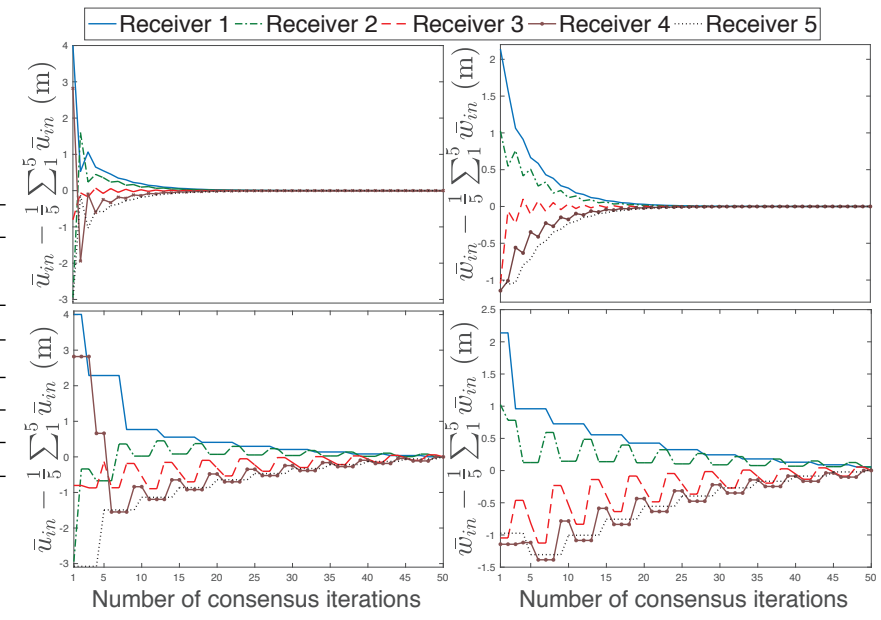

Fig. 2. The consensus process of a single target's location based on different schemes

performance of the proposed EMpLaR algorithm is portrayed after running $50 \mathrm{EM}$ iterations.

We verify the convergence speed of the proposed distributed EMpLaR algorithm in Sec. IV-B. The consensus impact of parameters $\bar{u}_{i, n}$ and $\bar{w}_{i, n}$ of a single target location based on the metropolis consensus [40] is depicted in Fig. ?? in a single simulation trail. For comparison, the consensus process based on broadcast gossip [42] with the update rate 0.5 is also characterized. The gossip algorithm has the advantage of simpler update rules. However, since the gossip algorithm only updates a pair of receivers at a consensus iteration, it suffers from a low convergence speed. It can be observed that for $p=50$, the gossip based algorithm cannot guarantee convergence.

Naturally, both the communications overhead and the energy consumption will increase as $N_{c}$ becomes larger. We compare the performance of the distributed EMpLaR algorithm for different $N_{c}$ values in Fig. 3 using the cumulative distribution function $(\mathrm{CDF})$ of the localization error. It can be observed that the performance gap between $N_{c}=25$ and $N_{c}=50$ is small, which was indeed expected based on the convergence behavior illustrated in Fig. 2. Therefore, $N_{c}=25$ can be adopted for reducing the computational complexity and the 


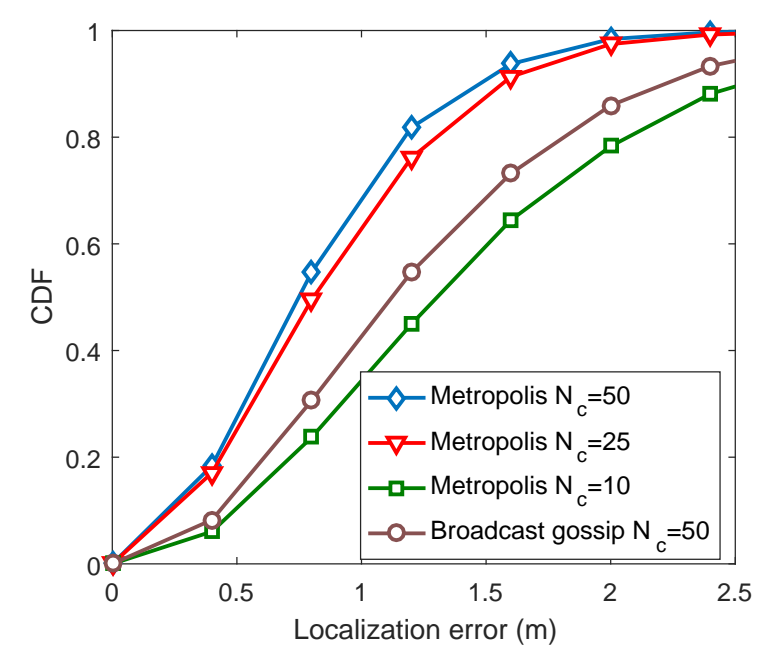

Fig. 3. Impact of the number of iterations for average consensus

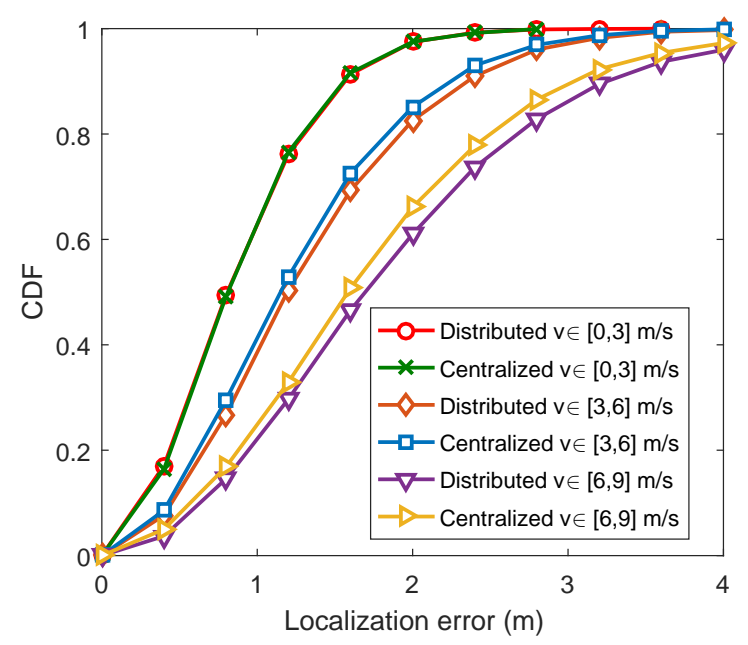

Fig. 4. The impact of delay introduced by EM and consensus iterations at different moving velocity of targets

communications overhead.

Next, we evaluate the impact of delay imposed by the iterations on the performance of our EMpLaR algorithm. Since the targets are moving, the information used in the iterative processing may become out-of-date, hence imposing a performance loss. In Fig. 4, the CDFs of the proposed centralized and distributed algorithm's localization error for different velocities is plotted. ${ }^{7}$ We can see that for higher velocity, both algorithms suffer from a performance degradation. Due to the extra delay imposed on the consensus process, the performance gap between the centralized and distributed EMpLaR algorithms becomes larger.

The number of measurements is related both to the number of targets and to that of the receivers. According to Section V, more measurements will increase the Fisher information and hence improve the performance. Fig. 5 shows localization error of the proposed algorithm versus the measurement noise for different number of targets. It is seen that the localization

\footnotetext{
${ }^{7}$ We assume in a single time slot, the targets move with constant velocity. A time division multiple access protocol is considered in the consensus process, which will introduce delay in each EM iteration, e.g., $20 \mathrm{~ms}$ in this simulation.
}

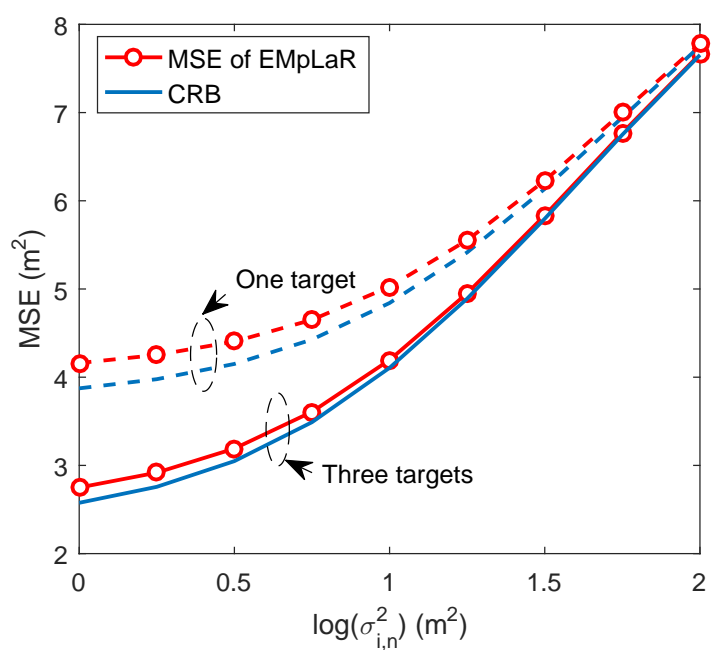

Fig. 5. MSE of the proposed algorithm with different number of targets

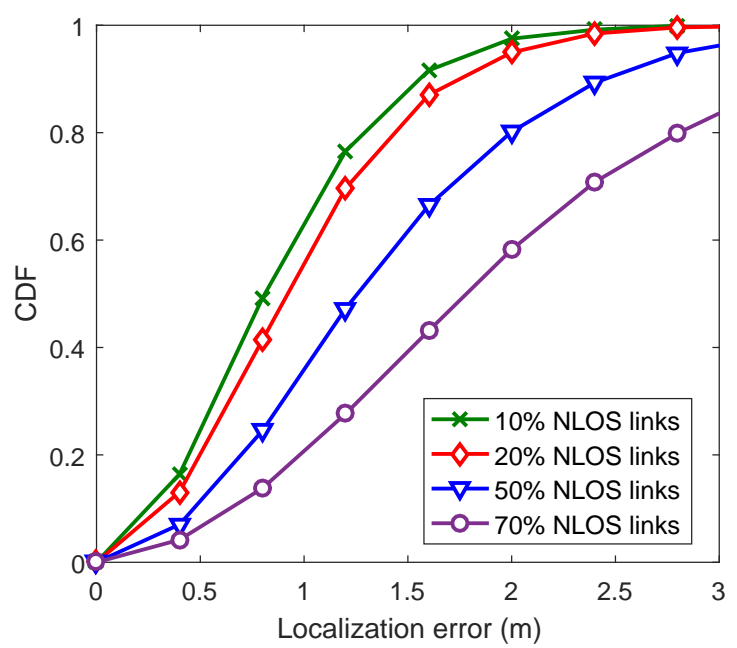

Fig. 6. CDF of the proposed algorithm with different percentage of NLOS links

accuracy improves with the number of targets. This can also be observed from (19) since more targets will improve the clock estimation accuracy and in turn, the localization result becomes more accurate.

In Fig. 6, the CDF of the proposed algorithm's localization error is illustrated for different percentages of NLOS links. It is seen that the proposed EMpLaR algorithm is capable of handling a practical mixed LOS/NLOS environment. However, for a higher percentage of NLOS links, the performance of the proposed algorithm becomes degraded. In Fig. 7, we illustrate the MSE of target locations versus the time slot index to evaluate the tracking performance of the proposed algorithm. We can observe the centralized algorithm converges in 5 seconds. Due to the delay caused by distributed processing, the distributed algorithm converges a little slower than the centralized one. Nevertheless, both algorithms can reach convergence in 7 seconds. In Fig. 8, the true trajectory of a target and the estimated trajectories based on the proposed centralized and distributed EMpLaR algorithms are plotted. We can see the estimated trajectories are close to the true trajectory after several time slots. 


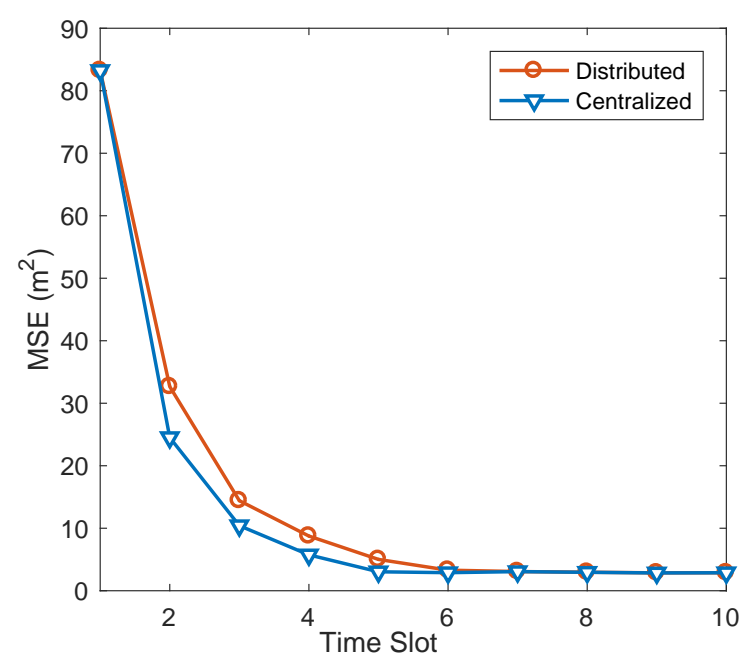

Fig. 7. MSE of the proposed algorithm versus time

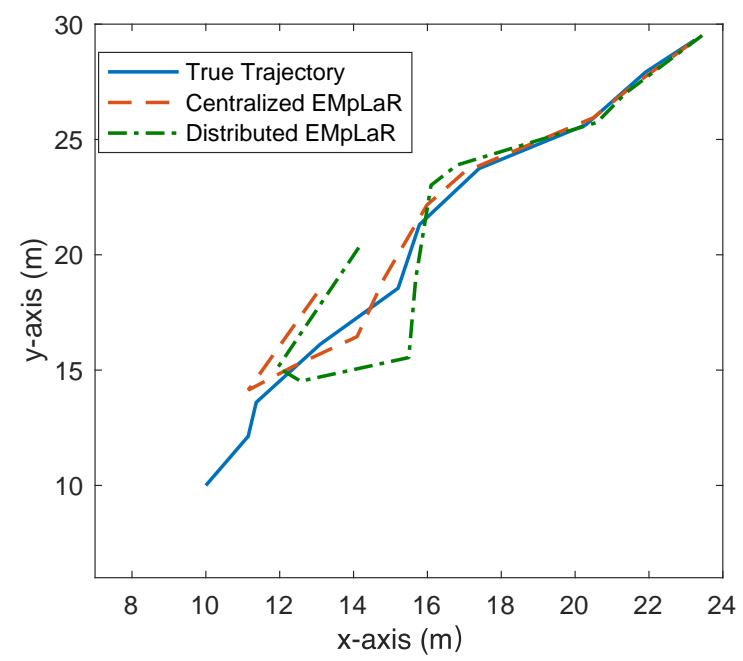

Fig. 8. The estimated trajectories based on the proposed algorithms and the true trajectory

\section{Comparison with State-of-the-art Algorithms}

In Fig. 9, we compare the proposed EMpLaR method to the state-of-the-art algorithms. It is observed that in an asynchronous network, the two step estimation (TSE) method of [24] which ignores the clock offsets suffers from a significant performance degradation. For the extended Kalman filter $(\mathrm{EKF})$, the direct linearization of the range measurement function in (6) leads to a substantial performance loss compared to both our numerical and Taylor expansion based methods. Moreover, due to the presence of clock offsets $\boldsymbol{\theta}$, extending the EKF to a distributed scenario is not straightforward [43]. The proposed centralized and distributed EMpLaR algorithms outperform both the TSE and EKF methods. As a benchmark, our centralized EMpLaR algorithm relying on numerical method in the M-step and our distributed likelihood consensus algorithm with distributed particle filtering (DPF) are also plotted. We can observe that the distributed EMpLaR performs similarly to the centralized EMpLaR using the first-order Taylor expansion, which verified the benefits of our quadratic polynomial approximation and of the average

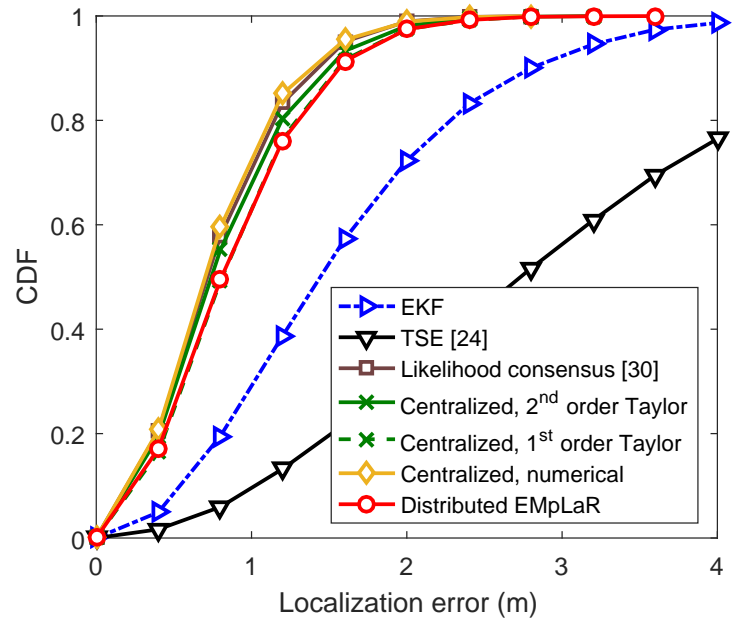

Fig. 9. Performance comparison with state-of-the-art algorithms

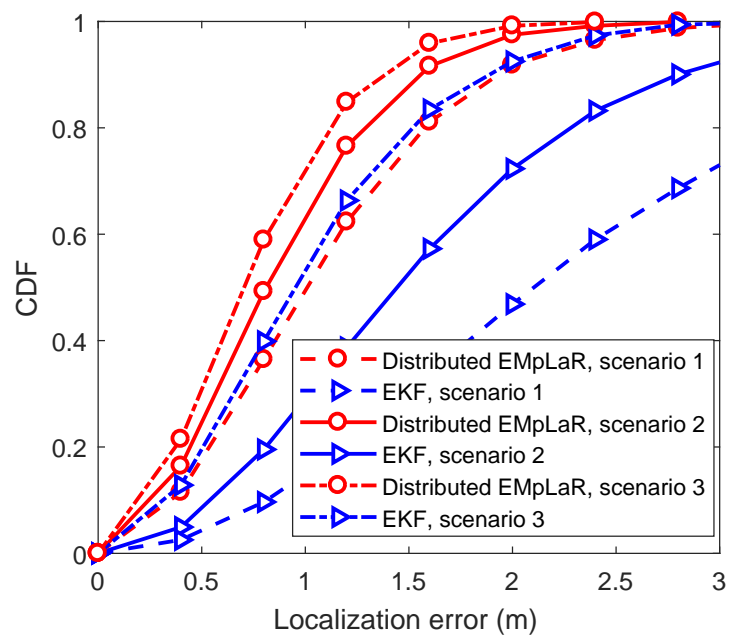

Fig. 10. Comparison of TSE method and the proposed algorithm with different level of clock offsets

consensus employing our distributed implementation.

The level of clock offsets may vary depending on the accuracy of coarse synchronization method. We compare the proposed EMpLaR algorithms to the EKF method in three scenarios in conjunction with different levels of clock offsets: (i) low accuracy: $\sigma_{\theta_{n}}=0.5 \mathrm{~ms}$; (ii) medium accuracy: $\sigma_{\theta_{n}}=$ 100ns; (iii) high accuracy: $\sigma_{\theta_{n}}=10 \mathrm{~ns}$. Since the centralized and distributed algorithm have similar localization accuracy, we only provide the results of the distributed EMpLaR for clarity. It is observed from Fig. 10 that for medium and large clock offset, the EKF method suffers significant performance losses and the proposed EMpLaR algorithms have to be employed.

Let us now characterize the MSE of the proposed EMpLaR algorithms versus the measurement noise in Fig. 11. The TSE and TDOA based methods are also included for comparison. Due to the noise enhancement imposed by the differential processing invoked for TDOA measurements, the TDOA method suffers from a significant performance loss in the high measurement noise region. On the other hand, for a low measurement noise, the gap between the TSE 


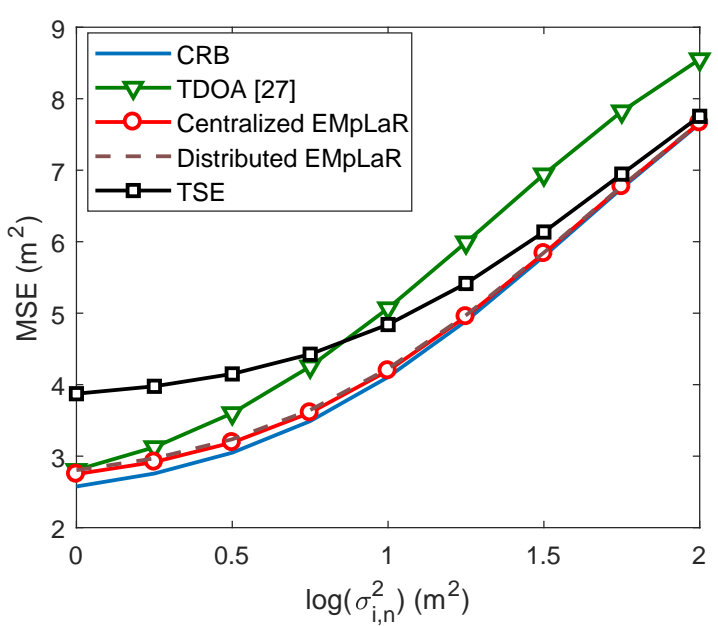

Fig. 11. MSE of the TSE, TDOA and the proposed EMpLaR algorithm versus measurement noise

TABLE II

Computational Complexity of Centralized Method

\begin{tabular}{|l|c|r|}
\hline Algorithm & Complexity & Execution time \\
\hline EKF & $\mathcal{O}\left((2 T+A)^{3}\right)$ & $0.623 \mathrm{~s}$ \\
\hline Numerical EMpLaR & $\mathcal{O}(K L T A)$ & $1.321 \mathrm{~s}$ \\
\hline Taylor expansion-based EMpLaR & $\mathcal{O}(L T A)$ & $0.026 \mathrm{~s}$ \\
\hline
\end{tabular}

method and the CRB increases, since the clock offsets begin to govern the performance loss. It can be observed that both the centralized and the distributed EMpLaR algorithms are capable of approaching the CRB and outperform both the TSE as well as TDOA methods.

\section{Computational Complexity and Communications Overhead Analysis}

We first compare the computational complexity of the centralized algorithms ${ }^{8}$, which is shown in Table II in terms of the number of multiplications. To distinguish the NLOS/LOS links, a complexity of $R_{N / L}$ is required. However, compared to the passive localization process, this complexity is rather limited and it is not considered here. The complexity of the proposed EMpLaR algorithm depends on the operations used in the M-step. For the Taylor expansion-based EMpLaR algorithm, the update rule is given in closed form, therefore its complexity is on the order of $\mathcal{O}(L T A)$. When the samplebased numerical method is employed in the M-step, the complexity becomes $\mathcal{O}(K L T A)$, where $K$ is the number of samples used. Hence, the proposed Taylor expansionbased EMpLaR algorithm may be deemed more attractive for practical applications.

TABLE III

COMmUnications OVERHEAD OF Distributed Algorithm

\begin{tabular}{|l|c|c|}
\hline Algorithm & Communications Overhead & Value \\
\hline DPF & $\mathcal{O}\left(J A N_{c}\right)$ & $J$ is large \\
\hline Distributed EMpLaR & $\mathcal{O}\left(3 A L N_{c}\right)$ & $L$ is small \\
\hline
\end{tabular}

In Table III, the communications overhead of DPF and of the proposed distributed EMpLaR algorithm are compared. For

\footnotetext{
${ }^{8}$ In the centralized methods, the measurements are collected in a fusion center. That is to say the communications overhead of all algorithms are identical.
}

the distributed EMpLaR, only three local parameters have to be broadcast to neighboring receivers. Since consensus should be reached at each EM iteration, the communications overhead of the distributed EMpLaR scales as $\mathcal{O}\left(3 A L N_{c}\right)$, where $L$ is the number of EM iterations, and $N_{c}$ is the number of consensus iterations. For the likelihood consensus based on DPF, all local receivers reach an agreement concerning the global likelihood function before EM iterations. The particles and their corresponding weights that are used to represent the likelihood functions have to be transmitted between receivers. Therefore, the communications overhead of DPF scales as $\mathcal{O}\left(J A N_{c}\right)$, where $J$ is the number of particles for the target coordinates and clock offsets. Generally, to provide accurate global likelihood functions, the number of particles is much higher than $3 L$. Overall, we can conclude that the distributed EMpLaR imposes a lower communications overhead than the conventional DPF.

\section{CONCLUSIONS}

We proposed expectation maximization based algorithms for the passive localization of asynchronous receivers. To obtain analytical solutions in the M-step, we invoked the classic Taylor expansion for approximating the nonlinear terms in the log augmented density of (15). As a result, the computational complexity became much lower than that of the numerical method. Furthermore, we considered a scenario operating without a fusion center and proposed a fully distributed algorithm based on average consensus. A quadratic polynomial was utilized for parameterizing the local functions. Consequently, only a few parameters had to be exchanged among the receivers in the consensus process. It was shown that the communications overhead of the proposed distributed EMpLaR algorithm is lower than that of the DPF-based likelihood consensus method. The Cramér-Rao bound of passive target location estimates was then derived as our benchmark of the proposed algorithms. Our simulation results showed that both the centralized and the distributed EMpLaR algorithms improve the performance of the conventional two-step estimation method and of the extended Kalman filter, and indeed they approach the CramérRao bound.

\section{APPENDIX A}

\section{DERIVATION OF COEFFICIENTS IN (24) AND (25) FOR THE FIRST-ORDER AND THE SECOND-ORDER TAYLOR EXPANSION}

We approximate the nonlinear terms $\left\|\mathbf{x}_{i}\right\|,\left\|\mathbf{x}_{i}-\boldsymbol{\kappa}_{n}\right\|$ and $\left\|\mathbf{x}_{i}-\boldsymbol{\kappa}_{n}\right\| \cdot\left\|\mathbf{x}_{i}\right\|$ based on the first-order and the secondorder Taylor series expansions at the previous target location estimate $\hat{\mathbf{x}}_{i}^{(l-1)}=\left[\hat{x}_{i}^{(l-1)}, \hat{y}_{i}^{(l-1)}\right]^{\mathrm{T}}$.

The first order Taylor expansion is given by

$$
\begin{gathered}
f\left(\mathbf{x}_{i}\right) \simeq f\left(\hat{\mathbf{x}}_{i}^{(l-1)}\right)+\frac{\partial f\left(\hat{\mathbf{x}}_{i}^{(l-1)}\right)}{\partial x_{i}}\left(x_{i}-\hat{x}_{i}^{(l-1)}\right) \\
+\frac{\partial f\left(\hat{\mathbf{x}}_{i}^{(l-1)}\right)}{\partial y_{i}}\left(y_{i}-\hat{y}_{i}^{(l-1)}\right) .
\end{gathered}
$$


Using (46) to expand $\left\|\mathbf{x}_{i}\right\|,\left\|\mathbf{x}_{i}-\boldsymbol{\kappa}_{n}\right\|$ and $\left\|\mathbf{x}_{i}-\boldsymbol{\kappa}_{n}\right\| \cdot\left\|\mathbf{x}_{i}\right\|$ and substituting the results into (15), the coefficients in (24) can be derived after some manipulations, i.e.,

$$
\begin{gathered}
A_{i}^{(l-1)}=C_{i}^{(l-1)}=1, \\
B_{i}^{(l-1)}=0,
\end{gathered}
$$

and

$D_{i}^{(l-1)}=\left(\sum_{n} \frac{1}{\sigma_{i, n}^{2}}+\frac{1}{\sigma_{v_{x}}^{2}}\right)^{-1}\left(\frac{\hat{x}_{i}^{(l-1)}+\delta_{t} \hat{v}_{x_{i}}^{k-1}}{\sigma_{v_{x}}^{2}}+\sum_{n} \frac{1}{\sigma_{i, n}^{2}}\right.$

$\left(\frac{a_{n}}{2}+\frac{R_{i, n}-v m_{\theta_{n}}^{(l)}-\hat{d}_{i, n}^{(l-1)}}{2} \frac{\hat{x}_{i}^{(l-1)}}{\hat{d}_{i}^{(l-1)}}+\frac{R_{i, n}-v m_{\theta_{n}}^{(l)}-\hat{d}_{i}^{(l-1)}}{2} C_{i}^{(l-1)}=\sum_{n} \frac{1}{\sigma_{i, n}^{2}}\left(\frac{\hat{x}_{i}^{(l-1)}\left(\hat{d}_{i, n}^{(l-1)}\right)^{3}+\left(\hat{x}_{i}^{(l-1)}-a_{n}\right)\left(\hat{d}_{i}^{(l-1)}\right)^{3}}{\left(\hat{d}_{i}^{(l-1)} \hat{d}_{i, n}^{(l-1)}\right)^{2}}\right.\right.$

$\left.\left.\times \frac{\hat{x}_{i}^{(l-1)}-a_{n}}{\hat{d}_{i, n}^{(l-1)}}\right)-\sum_{(i, n) \in \mathcal{C}^{\mathrm{NL} O S}} \frac{m_{\beta_{i, n}}^{(l)}}{2 \sigma_{i, n}^{2}}\left(\frac{\hat{x}_{i}^{(l-1)}}{\hat{d}_{i}^{(l-1)}}+\frac{\hat{x}_{i}^{(l-1)}-a_{n}}{\hat{d}_{i, n}^{(l-1)}}\right)\right)$,

and

$E_{i}^{(l-1)}=\left(\sum_{n} \frac{1}{\sigma_{i, n}^{2}}+\frac{1}{\sigma_{v_{y}}^{2}}\right)^{-1}\left(\frac{\hat{y}_{i}^{(l-1)}+\delta_{t} \hat{v}_{y_{i}}^{k-1}}{\sigma_{v_{y}}^{2}}+\sum_{n} \frac{1}{\sigma_{i, n}^{2}}\right.$

$\left(\frac{b_{n}}{2}+\frac{R_{i, n}-v m_{\theta_{n}}^{(l)}-\hat{d}_{i, n}^{(l-1)}}{2} \frac{\hat{y}_{i}^{(l-1)}}{\hat{d}_{i}^{(l-1)}}+\frac{R_{i, n}-v m_{\theta_{n}}^{(l)}-\hat{d}_{i}^{(l-1)}}{2}\right.$

$\left.\left.\times \frac{\hat{y}_{i}^{(l-1)}-b_{n}}{\hat{d}_{i, n}^{(l-1)}}\right)-\sum_{(i, n) \in \mathcal{C}^{\mathrm{NLOS}}} \frac{m_{\beta_{i, n}}^{(l)}}{2 \sigma_{i, n}^{2}}\left(\frac{\hat{y}_{i}^{(l-1)}}{\hat{d}_{i}^{(l-1)}}+\frac{\hat{y}_{i}^{(l-1)}-b_{n}}{\hat{d}_{i, n}^{(l-1)}}\right)\right)$,

with $\hat{d}_{i}^{(l-1)} \triangleq\left\|\hat{\mathbf{x}}_{i}^{(l-1)}\right\|$ and $\hat{d}_{i, n}^{(l-1)} \triangleq\left\|\boldsymbol{\kappa}_{n}-\hat{\mathbf{x}}_{i}^{(l-1)}\right\|$.

Similarly, using the second order Taylor expansion, which is given by

$f\left(\mathbf{x}_{i}\right) \simeq$

$f\left(\hat{\mathbf{x}}_{i}^{(l-1)}\right)+\frac{\partial f\left(\hat{\mathbf{x}}_{i}^{(l-1)}\right)}{\partial x_{i}}\left(x_{i}-\hat{x}_{i}^{(l-1)}\right)+\frac{\partial f\left(\hat{\mathbf{x}}_{i}^{(l-1)}\right)}{\partial y_{i}}\left(y_{i}-\hat{y}_{i}^{(l-1)}\right)$ $+\frac{1}{2} \frac{\partial^{2} f\left(\hat{\mathbf{x}}_{i}^{(l-1)}\right)}{\partial x_{i}^{2}}\left(x_{i}-\hat{x}_{i}^{(l-1)}\right)^{2}+\frac{1}{2} \frac{\partial^{2} f\left(\hat{\mathbf{x}}_{i}^{(l-1)}\right)}{\partial y_{i}^{2}}\left(y_{i}-\hat{y}_{i}^{(l-1)}\right)^{2}$

$$
+\frac{1}{2} \frac{\partial^{2} f\left(\hat{\mathbf{x}}_{i}^{(l-1)}\right)}{\partial x_{i} \partial y_{i}}\left(x_{i}-\hat{x}_{I}^{(l-1)}\right)\left(y_{i}-\hat{y}_{i}^{(l-1)}\right)
$$

the coefficients in (24) can be derived as follows

$$
\begin{aligned}
A_{i}^{(l-1)}= & \sum_{n} \frac{1}{\sigma_{i, n}^{2}}\left(\frac{\hat{x}_{i}^{(l-1)}\left(\hat{d}_{i, n}^{(l-1)}\right)^{3}+\left(\hat{x}_{i}^{(l-1)}-a_{n}\right)\left(\hat{d}_{i}^{(l-1)}\right)^{3}}{\left(\hat{d}_{i}^{(l-1)} \hat{d}_{i, n}^{(l-1)}\right)^{2}}\right. \\
+ & \left.2+\frac{\left(\hat{d}_{i}^{(l-1)}\right)^{2}+\left(\hat{d}_{i, n}^{(l-1)}\right)^{2}+4 \hat{x}_{i}^{(l-1)}\left(\hat{x}_{i}^{(l-1)}-a_{n}\right)}{\hat{d}_{i}^{(l-1)} \hat{d}_{i, n}^{(l-1)}}\right) \\
+ & \left(\sum_{(i, n) \in \mathcal{C}^{\mathrm{LOS}}}\left(v m_{\theta_{n}}^{(l)}-R_{i, n}\right)+\sum_{(i, n) \in \mathcal{C}^{\mathrm{NL} O S}}\left(m_{\beta_{i, n}}^{(l)}+v m_{\theta_{n}}^{(l)}-R_{i, n}\right)\right) \\
& {\left[\frac{\left(\hat{y}_{i}^{(l-1)}\right)^{2}}{\sigma_{i, n}^{2}\left(\hat{d}_{i}^{(l-1)}\right)^{3}}+\frac{\left(\hat{y}_{i}^{(l-1)}-b_{n}\right)^{2}}{\sigma_{i, n}^{2}\left(\hat{d}_{i, n}^{(l-1)}\right)^{3}}\right]+\frac{1}{\sigma_{v_{y}}^{2}}, }
\end{aligned}
$$

$$
\begin{aligned}
B_{i}^{(l-1)}= & \sum_{n} \frac{\hat{x}_{i}^{(l-1)}\left(\hat{y}_{i}^{(l-1)}-b_{n}\right)+\hat{y}_{i}^{(l-1)}\left(\hat{x}_{i}^{(l-1)}-a_{n}\right)}{\sigma_{i, n}^{2} \hat{d}_{i}^{(l-1)} \hat{d}_{i, n}^{(l-1)}} \\
+ & \left(\sum_{(i, n) \in \mathcal{C}^{\mathrm{LOS}}}\left(v m_{\theta_{n}}^{(l)}-R_{i, n}\right)+\sum_{(i, n) \in \mathcal{C}^{\mathrm{NLOS}}}\left(m_{\beta_{i, n}}^{(l)}+v m_{\theta_{n}}^{(l)}-R_{i, n}\right)\right) \\
& {\left[\frac{\left(\hat{x}_{i}^{(l-1)}-a_{n}\right)^{2}\left(\hat{y}_{i}^{(l-1)}-b_{n}\right)-\left(\hat{y}_{i}^{(l-1)}-b_{n}\right)^{3}}{\sigma_{i, n}^{2}\left(\hat{d}_{i, n}^{(l-1)}\right)^{4}}\right.}
\end{aligned}
$$$$
\left.+\frac{\left(\hat{x}_{i}^{(l-1)}\right)^{2} \hat{y}_{i}^{(l-1)}-\left(\hat{y}_{i}^{(l-1)}\right)^{3}}{\sigma_{i, n}^{2}\left(\hat{d}_{i}^{(l-1)}\right)^{3}}\right],
$$$$
\left.+2+\frac{\left(\hat{d}_{i}^{(l-1)}\right)^{2}+\left(\hat{d}_{i, n}^{(l-1)}\right)^{2}+4 \hat{y}_{i}^{(l-1)}\left(\hat{y}_{i}^{(l-1)}-b_{n}\right)}{\hat{d}_{i}^{(l-1)} \hat{d}_{i, n}^{(l-1)}}\right)
$$$$
+\left(\sum_{(i, n) \in \mathcal{C}^{\mathrm{LOS}}}\left(v m_{\theta_{n}}^{(l)}-R_{i, n}\right)+\sum_{(i, n) \in \mathcal{C}^{\mathrm{NLOS}}}\left(m_{\beta_{i, n}}^{(l)}+v m_{\theta_{n}}^{(l)}-R_{i, n}\right)\right)
$$

$$
D_{i}^{(l-1)}=\sum_{n} \frac{1}{\sigma_{i, n}^{2}}\left(\frac{\hat{x}_{i}^{(l-1)}\left(\hat{d}_{i, n}^{(l-1)}\right)^{2}+\left(\hat{x}_{i}^{(l-1)}-a_{n}\right)\left(\hat{d}_{i}^{(l-1)}\right)^{2}}{\hat{d}_{i}^{(l-1)} \hat{d}_{i, n}^{(l-1)}}\right.
$$$$
-\hat{x}_{i}^{(l-1)} \frac{\hat{x}_{i}^{(l-1)}\left(\hat{d}_{i, n}^{(l-1)}\right)^{3}+\left(\hat{x}_{i}^{(l-1)}-a_{n}\right)\left(\hat{d}_{i}^{(l-1)}\right)^{3}}{\left(\hat{d}_{i}^{(l-1)} \hat{d}_{i, n}^{(l-1)}\right)^{2}}-a_{n}
$$$$
\left.-\hat{y}_{i}^{(l-1)} \frac{\hat{x}_{i}^{(l-1)}\left(\hat{y}_{i}^{(l-1)}-b_{n}\right)+\hat{y}_{i}^{(l-1)}\left(\hat{x}_{i}^{(l-1)}-a_{n}\right)}{\left(\hat{d}_{i}^{(l-1)} \hat{d}_{i, n}^{(l-1)}\right)}\right)
$$$$
+\left(\sum_{(i, n) \in \mathcal{C}^{\mathrm{LOS}}} \frac{v m_{\theta_{n}}^{(l)}-R_{i, n}}{\sigma_{i, n}^{2}}+\sum_{(i, n) \in \mathcal{C}^{\mathrm{NLOS}}} \frac{m_{\beta_{i, n}}^{(l)}+v m_{\theta_{n}}^{(l)}-R_{i, n}}{\sigma_{i, n}^{2}}\right)
$$$$
\left[\frac{\hat{x}_{i}^{(l-1)}}{\hat{d}_{i}^{(l-1)}}-\hat{x}_{i}^{(l-1)} \frac{\left(\hat{y}_{i}^{(l-1)}\right)^{2}}{\left(\hat{d}_{i}^{(l-1)}\right)^{3}}+\frac{\hat{x}_{i}^{(l-1)}-a_{n}}{\hat{d}_{i, n}^{(l-1)}}\right.
$$$$
\left.-\hat{x}_{i}^{(l-1)} \frac{\left(\hat{y}_{i}^{(l-1)}-b_{n}\right)^{2}}{\left(\hat{d}_{i, n}^{(l-1)}\right)^{3}}\right]+\frac{\hat{x}_{i}^{(l-1)}+\delta_{t} \hat{v}_{x_{i}}^{k-1}}{\sigma_{v_{x}}^{2}},
$$

$$
\begin{aligned}
E_{i}^{(l-1)} & =\sum_{n} \frac{1}{\sigma_{i, n}^{2}}\left(\frac{\hat{y}_{i}^{(l-1)}\left(\hat{d}_{i, n}^{(l-1)}\right)^{2}+\left(\hat{y}_{i}^{(l-1)}-b_{n}\right)\left(\hat{d}_{i}^{(l-1)}\right)^{2}}{\hat{d}_{i}^{(l-1)} \hat{d}_{i, n}^{(l-1)}}\right. \\
& -\hat{y}_{i}^{(l-1)} \frac{\hat{y}_{i}^{(l-1)}\left(\hat{d}_{i, n}^{(l-1)}\right)^{3}+\left(\hat{y}_{i}^{(l-1)}-b_{n}\right)\left(\hat{d}_{i}^{(l-1)}\right)^{3}}{\left(\hat{d}_{i}^{(l-1)} \hat{d}_{i, n}^{(l-1)}\right)^{2}}-b_{n}
\end{aligned}
$$$$
\left.-\hat{x}_{i}^{(l-1)} \frac{\hat{x}_{i}^{(l-1)}\left(\hat{y}_{i}^{(l-1)}-b_{n}\right)+\hat{y}_{i}^{(l-1)}\left(\hat{x}_{i}^{(l-1)}-a_{n}\right)}{\left(\hat{d}_{i}^{(l-1)} \hat{d}_{i, n}^{(l-1)}\right)}\right)
$$$$
+\left(\sum_{(i, n) \in \mathcal{C}^{\operatorname{LOS}}} \frac{v m_{\theta_{n}}^{(l)}-R_{i, n}}{\sigma_{i, n}^{2}}+\sum_{(i, n) \in \mathcal{C}^{\mathrm{NLOS}}} \frac{m_{\beta_{i, n}}^{(l)}+v m_{\theta_{n}}^{(l)}-R_{i, n}}{\sigma_{i, n}^{2}}\right)
$$$$
\left[\frac{\hat{y}_{i}^{(l-1)}}{\hat{d}_{i}^{(l-1)}}-\hat{y}_{i}^{(l-1)} \frac{\left(\hat{x}_{i}^{(l-1)}\right)^{2}}{\left(\hat{d}_{i}^{(l-1)}\right)^{3}}+\frac{\hat{y}_{i}^{(l-1)}-b_{n}}{\hat{d}_{i, n}^{(l-1)}}\right.
$$$$
\left.-\hat{y}_{i}^{(l-1)} \frac{\left(\hat{x}_{i}^{(l-1)}-a_{n}\right)^{2}}{\left(\hat{d}_{i, n}^{(l-1)}\right)^{3}}\right]+\frac{\hat{y}_{i}^{(l-1)}+\delta_{t} \hat{v}_{y_{i}}^{k-1}}{\sigma_{v_{y}}^{2}} .
$$ 


\section{REFERENCES}

[1] I. F. Akyildiz, W. Su, Y. Sankarasubramaniam, and E. Cayirci, "A survey on sensor networks," IEEE Commun. Mag., vol. 40, no. 8, pp. 102-114, 2002.

[2] N. Patwari, J. N. Ash, S. Kyperountas, A. O. Hero, R. L. Moses, and N. S. Correal, "Locating the nodes: cooperative localization in wireless sensor networks," IEEE Signal Process. Mag., vol. 22, no. 4, pp. 54-69, 2005.

[3] K.-F. Ssu, C.-H. Ou, and H. C. Jiau, "Localization with mobile anchor points in wireless sensor networks," IEEE Trans. Veh. Technol., vol. 54, no. 3, pp. 1187-1197, 2005.

[4] Y.-T. Chan, W.-Y. Tsui, H.-C. So, and P.-c. Ching, "Time-of-arrival based localization under NLOS conditions," IEEE Trans. Veh. Technol., vol. 55, no. 1, pp. 17-24, 2006.

[5] K. Yu, "3-D localization error analysis in wireless networks," IEEE Trans. Wireless Commun., vol. 6, pp. 3472-3481, 2007.

[6] A. J. Weiss, "On the accuracy of a cellular location system based on RSS measurements," IEEE Trans. Veh. Technol., vol. 52, no. 6, pp. 15081518, 2003.

[7] B. Friedlander, "A passive localization algorithm and its accuracy analysis," IEEE J. Ocean. Eng., vol. 12, no. 1, pp. 234-245, 1987.

[8] B. Etzlinger, C. Pimminger, S. Fischereder, and A. Springer, "Passive localization and synchronization in the presence of affine clocks," in Proc. 49th Asilomar Conf. Signals, Sys., Comput. IEEE, Oct. 2015, pp. 1655-1658.

[9] O. Jean and A. J. Weiss, "Passive localization and synchronization using arbitrary signals," IEEE Trans. Signal Process., vol. 62, no. 8, pp. 2143 2150, Apr. 2014.

[10] M. Leng and Y.-C. Wu, "On clock synchronization algorithms for wireless sensor networks under unknown delay," IEEE Trans. Veh. Technol., vol. 59, no. 1, pp. 182-190, 2010.

[11] Y.-C. Wu, Q. Chaudhari, and E. Serpedin, "Clock synchronization of wireless sensor networks," IEEE Signal Process. Mag., vol. 28, no. 1, pp. 124-138, 2011.

[12] B. Etzlinger, H. Wymeersch, and A. Springer, "Cooperative synchronization in wireless networks," IEEE Trans. Signal Process., vol. 62 no. 11, pp. 2837-2849, 2014

[13] Y. Xiong, N. Wu, Y. Shen, and M. Z. Win, "Cooperative network synchronization: Asymptotic analysis," IEEE Trans. Signal Process., vol. 66, no. 3, pp. 757-772, 2018.

[14] H. Wymeersch, J. Lien, and M. Z. Win, "Cooperative localization in wireless networks," Proc. IEEE, vol. 97, no. 2, pp. 427-450, 2009.

[15] V. Vivekanandan and V. W. Wong, "Concentric anchor beacon localization algorithm for wireless sensor networks," IEEE Trans. Veh. Technol., vol. 56, no. 5, pp. 2733-2744, 2007.

[16] H. Wymeersch, S. Maranò, W. M. Gifford, and M. Z. Win, "A machine learning approach to ranging error mitigation for UWB localization," IEEE Trans. Commun., vol. 60, no. 6, pp. 1719-1728, 2012.

[17] J. Teng, H. Snoussi, C. Richard, and R. Zhou, "Distributed variational filtering for simultaneous sensor localization and target tracking in wireless sensor networks," IEEE Trans. Veh. Technol., vol. 61, no. 5, pp. 2305-2318, 2012.

[18] W. Yuan, N. Wu, H. Wang, and J. Kuang, "Distributed passive localization with asynchronous receivers based on expectation maximization," in Proc. 2015 IEEE Global Telecommun. Conf. IEEE, 2015, pp. 1-6.

[19] J. Zheng and Y.-C. Wu, "Joint time synchronization and localization of an unknown node in wireless sensor networks," IEEE Trans. Signal Process., vol. 58, no. 3, pp. 1309-1320, 2010.

[20] J. Yan, X. Zhang, X. Luo, Y. Wang, C. Chen, and X. Guan, "Asynchronous localization with mobility prediction for underwater acoustic sensor networks," IEEE Trans. Veh. Technol., 2017.

[21] A. Ahmad, E. Serpedin, H. Nounou, and M. Nounou, "Joint node localization and time-varying clock synchronization in wireless sensor networks," IEEE Trans. Wireless Commun., vol. 12, no. 10, 2013.

[22] M. R. Gholami, S. Gezici, and E. G. Strom, “TDOA based positioning in the presence of unknown clock skew," IEEE Trans. Commun., vol. 61 , no. 6, pp. 2522-2534, 2013.

[23] W. Yuan, N. Wu, B. Etzlinger, H. Wang, and J. Kuang, "Cooperative joint localization and clock synchronization based on Gaussian message passing in asynchronous wireless networks," IEEE Trans. Veh. Technol., vol. 65, no. 9, pp. 7258-7273, 2016.

[24] J. Shen, A. F. Molisch, and J. Salmi, "Accurate passive location estimation using TOA measurements," IEEE Trans. Wireless Commun., vol. 11 , no. 6 , pp. 2182-2192, 2012
[25] S. Bartoletti and A. Conti, "Passive network localization via UWB wireless sensor radars: The impact of TOA estimation," in Proc. IEEE Int. Conf. Ultra-Wideband, Jul. 2011, pp. 258-262.

[26] Y. Wang, S. Ma, and C. Chen, "TOA-based passive localization in quasisynchronous networks," IEEE Commun. Lett., vol. 18, no. 4, pp. 592595, Apr. 2014.

[27] Y. Zhou, C. L. Law, Y. L. Guan, and F. Chin, "Indoor elliptical localization based on asynchronous UWB range measurement," IEEE Trans. Instrum. Meas., vol. 60, no. 1, pp. 248-257, 2011.

[28] R. Olfati-Saber, E. Franco, E. Frazzoli, and J. S. Shamma, "Belief consensus and distributed hypothesis testing in sensor networks," in Networked Embedded Sensing and Control. Springer, 2006, pp. 169182.

[29] F. Meyer, O. Hlinka, H. Wymeersch, E. Riegler, and F. Hlawatsch, "Cooperative simultaneous localization and tracking in mobile agent networks," arXiv preprint arXiv:1403.1824, 2014

[30] O. Hlinka, F. Hlawatsch, and P. M. Djuric, "Distributed particle filtering in agent networks: A survey, classification, and comparison," IEEE Signal Process. Mag., vol. 30, no. 1, pp. 61-81, 2013.

[31] V. Savic, H. Wymeersch, and S. Zazo, "Belief consensus algorithms for fast distributed target tracking in wireless sensor networks," Signal Process., vol. 95, pp. 149-160, 2014.

[32] S. Venkatraman, J. Caffery, and H.-R. You, "A novel TOA location algorithm using LOS range estimation for nlos environments," IEEE Trans. Veh. Technol., vol. 53, no. 5, pp. 1515-1524, 2004

[33] A. P. Dempster, N. M. Laird, and D. B. Rubin, "Maximum likelihood from incomplete data via the EM algorithm," in J. Royal Stat. Soc., Ser. $B, 1977$, pp. 1-38.

[34] M. Leng and Y.-C. Wu, "Localization of wireless sensor nodes with erroneous anchors via EM algorithm," in IEEE Proc. Global Telecommun. Conf. IEEE, 2010, pp. 1-5.

[35] L. M. Ni, D. Zhang, and M. R. Souryal, "RFID-based localization and tracking technologies," IEEE Wireless Commun., vol. 18, no. 2, pp. 45$51,2011$.

[36] S. Marano, W. M. Gifford, H. Wymeersch, and M. Z. Win, "NLOS identification and mitigation for localization based on UWB experimental data," IEEE J. Sel. Areas Commun., vol. 28, no. 7, pp. 1026-1035, 2010.

[37] S. Venkatesh and R. M. Buehrer, "NLOS mitigation using linear programming in ultrawideband location-aware networks," IEEE Trans. Veh. Technol., vol. 56, no. 5, pp. 3182-3198, 2007.

[38] A. Logothetis and V. Krishnamurthy, "Expectation maximization algorithms for MAP estimation of jump Markov linear systems," IEEE Trans. Signal Process., vol. 47, no. 8, pp. 2139-2156, 1999.

[39] J. Shen and A. F. Molisch, "Discerning direct and indirect paths: Principle and application in passive target positioning systems," in Proc. IEEE Global Telecommun. Conf. (GLOBECOM 2011). IEEE, 2011 pp. 1-6.

[40] O. Hlinka, O. Sluciak, F. Hlawatsch, P. M. Djuric, and M. Rupp, "Likelihood consensus and its application to distributed particle filtering," IEEE Trans. Signal Process., vol. 60, no. 8, pp. 4334-4349, 2012.

[41] Y. Shen and M. Z. Win, "Fundamental limits of wideband localizationtpart I: A general framework," IEEE Trans. Inf. Theory, vol. 56, no. 10, pp. 4956-4980, 2010

[42] T. C. Aysal, M. E. Yildiz, A. D. Sarwate, and A. Scaglione, "Broadcast gossip algorithms for consensus," IEEE Trans. Signal Process., vol. 57, no. 7, pp. 2748-2761, 2009.

[43] R. Olfati-Saber, "Distributed kalman filtering for sensor networks," in Proc. IEEE Conf. Decision and Control,. IEEE, Feb. 2007, pp. 54925498.

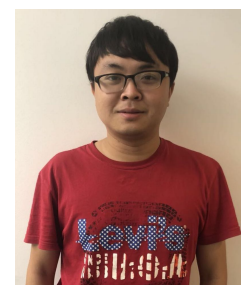

Weijie Yuan (S'15) received the B.S. degree from the Beijing Institute of Technology, Beijing, China, in 2013. He is currently pursuing the Ph.D. degree in Beijing Institute of Technology and University of Technology Sydney. He also serves as the research assistant in the University of Sydney and the visiting associate fellow in the University of Wollongong, In 2016, he was a Visiting Ph.D. Student with the Institute of Telecommunications, Vienna University of Technology, Austria. His research interests include statistical inference on graphical models. He has served as a TPC Member for several conferences. 


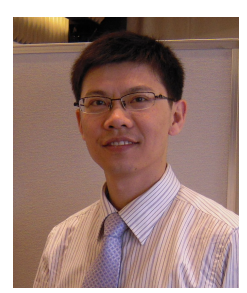

Nan Wu (M'11) received the B.S., M.S., and Ph.D degrees from the Beijing Institute of Technology (BIT), Beijing, China, in 2003, 2005, and 2011, respectively. From 2008 to 2009 , he was a Visiting Ph.D. Student with the Department of Electrical Engineering, Pennsylvania State University, USA. $\mathrm{He}$ is currently an Associate Professor with the School of Information and Electronics, BIT. His research interests include signal processing in wireless communication networks. He was a recipient of the National Excellent Doctoral Dissertation Award by MOE of China in 2013. He serves as an Associate Editor of IEEE ACCESS, International Journal of Electronics and Communications, KSII Trans- actions on Internet and Information Systems, and IEICE Transactions on Communications.

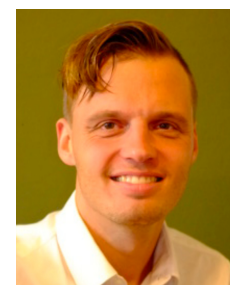

Bernhard Etzlinger (S'11-M'16) received the Dipl.-Ing. (M.Sc.) degree in mechatronics in 2010 and the Dr. techn. (Ph.D.) degree in technical sciences in 2016, both from Johannes Kepler University Linz, Linz, Austria. In 2010, he worked at Fraunhofer FKIE, Wachtberg, Germany. During his Ph.D. studies, he was a visiting student with the Department of Signals and Systems, Chalmers University of Technology, Gothenburg, Sweden, with the Department of Electrical and Computer Engineering, Texas A\&M University, College Station, TX, USA, and with the Ming Hsieh Department of Electrical Engineering, University of Southern California, CA, USA. His research activities are focused on statistical signal processing for receiver design and cooperative network clock synchronization and localization.

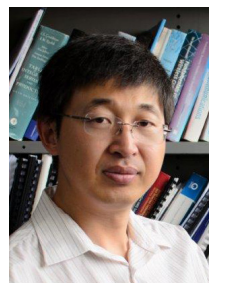

Yonghui Li (M'04-SM'09) received his PhD degree in November 2002 from Beijing University of Aeronautics and Astronautics. From 1999 2003, he was affiliated with Linkair Communication Inc, where he held a position of project manager with responsibility for the design of physical layer solutions for the LAS-CDMA system. Since 2003, he has been with the Centre of Excellence in Telecommunications, the University of Sydney, Australia. He is now a Professor in School of Electrical and Information Engineering, University of Sydney. He is the recipient of the Australian Queen Elizabeth II Fellowship in 2008 and the Australian Future Fellowship in 2012.

His current research interests are in the area of wireless communications, with a particular focus on MIMO, millimeter wave communications, machine to machine communications, coding techniques and cooperative communications. He holds a number of patents granted and pending in these fields. He is now an editor for IEEE transactions on communications and IEEE transactions on vehicular technology. He also served as a guest editor for several special issues of IEEE journals, such as IEEE JSAC special issue on Millimeter Wave Communications. He received the best paper awards from IEEE International Conference on Communications (ICC) 2014, IEEE PIMRC 2017 and IEEE Wireless Days Conferences (WD) 2014.

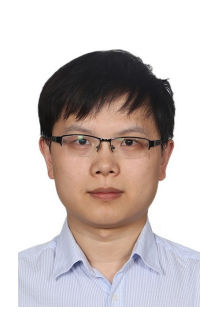

Chaoxing Yan (S'09-M'12) received the Bachelor degree in Electronics from Qingdao University, China, in 2006, and the Ph.D. degree in Communication and Information System from Beijing Institute of Technology (BIT), China, in 2012. Since March 2012, he has been with the Beijing Research Institute of Telemetry (BRIT), where he is now a Senior Engineer. From July 2017 to December 2017, he was a visiting scholar with the School of Electronics and Computer Science, the Univeristy of Southampton,

UK. He is the author of several scientific papers and more than 20 patents. He serves as Session Chairman of ICCC 2018. His main research interests are in the area of UAV datalink and satellite communication networking.

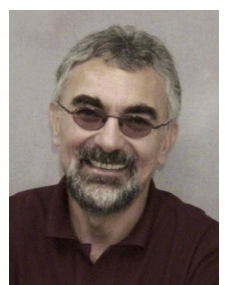

Lajos Hanzo (M'91-SM'92-F'04) (http://www. mobile.ecs soton.ac.uk) FREng, FIEEE, FIET, Fellow of EURASIP, DSc received his degree in electronics in 1976 and his doctorate in 1983. In 2009 he was awarded an honorary doctorate by the Technical University of Budapest and in 2015 by the University of Edinburgh. In 2016 he was admitted to the Hungarian Academy of Science. During his 40-year career in telecommunications he has held various research and academic posts in Hungary, Germany and the UK. Since 1986 he has been with the School of Electronics and Computer Science, University of Southampton, UK, where he holds the chair in telecommunications. He has successfully supervised $111 \mathrm{PhD}$ students, co-authored 18 John Wiley/IEEE Press books on mobile radio communications totalling in excess of 10000 pages, published 1772 research contributions at IEEE Xplore, acted both as TPC and General Chair of IEEE conferences, presented keynote lectures and has been awarded a number of distinctions. Currently he is directing an academic research team, working on a range of research projects in the field of wireless multimedia communications sponsored by industry, the Engineering and Physical Sciences Research Council (EPSRC) UK, the European Research Councils Advanced Fellow Grant and the Royal Societys Wolfson Research Merit Award. He is an enthusiastic supporter of industrial and academic liaison and he offers a range of industrial courses. He is also a Governor of the IEEE ComSoc and VTS. During 2008 - 2012 he was the Editor-in-Chief of the IEEE Press and a Chaired Professor also at Tsinghua University, Beijing. For further information on research in progress and associated publications please refer to http://www-mobile.ecs.soton.ac.uk 